\title{
Computer-Assisted Reading and Discovery for Student-Generated Text in Massive Open Online Courses
}

\author{
Justin Reich, Dustin Tingley, Jetson Leder-Luis, Margaret E. Roberts, Brandon M. Stewart \\ Harvard University, USA \\ justin_reich@harvard.edu
}

\begin{abstract}
Dealing with the vast quantities of text that students generate in Massive Open Online Courses (MOOCs) and other large-scale online learning environments is a daunting challenge. Computational tools are needed to help instructional teams uncover themes and patterns as students write in forums, assignments, and surveys. This paper introduces to the learning analytics community the Structural Topic Model, an approach to language processing that can 1) find syntactic patterns with semantic meaning in unstructured text, 2) identify variation in those patterns across covariates, and 3) uncover archetypal texts that exemplify the documents within a topical pattern. We show examples of computationally aided discovery and reading in three MOOC settings: mapping students' self-reported motivations, identifying themes in discussion forums, and uncovering patterns of feedback in course evaluations.
\end{abstract}

KEYWORDS: Massive Open Online Courses, topic modelling, text analysis, computer-assisted reading

\section{OVERVIEW}

Educators are constantly asking their students to write. They articulate needs and motivations in precourse surveys, communicate and collaborate in forums, demonstrate their understanding in assignments, and offer feedback about instructional approaches in course evaluations. In classes with low student-instructor ratios, instructional teams of faculty and teaching assistants can read, process, and provide feedback on the entire corpus of text produced by students. In large-scale learning environments, such as Massive Open Online Courses (MOOCs), there is far too much for instructors to read and process in a timely fashion. Consider two sources of student text: surveys and discussion forums. In the first year of operation, hundreds of thousands of students signed up for HarvardX courses on the edX platform, and they submitted over 240,000 answers to the open-response survey question: "Please share your reasons for signing up for edX." In the inaugural MITx class, the discussion forums included over 12,000 threads and nearly 100,000 individual posts (Breslow, Pritchard, DeBoer, Stump, \& Ho, 2013). These corpora represent two troves of important data. Understanding what motivates students to sign up for courses can help course developers tailor instruction to their students. Discussion forums are central sites for advancing student learning in many MOOCs, especially in the professions (Fisher, 2014; Reich et al., 2014a) and humanities (Reich et al., 2014b), but these spaces can rapidly become overwhelming to follow or analyze, especially for faculty discovering the arduous demands of teaching a MOOC (Grainger, 2013; Kolowich, 2014). 
(2015). Computer-Assisted Reading and Discovery for Student-Generated Text in Massive Open Online Courses. Journal of Learning Analytics, 2(1), 156-184.

In this paper, we introduce advances in computer-assisted techniques for discovery and analysis of student-produced text, and we illustrate these techniques with examples from MOOC pre-course surveys, discussion forum threads, and course evaluations. We demonstrate a method of conducting text analysis known as the Structural Topic Model (STM) (Lucas et al., 2013; Roberts, Stewart, Tingley \& Airoldi, 2013; Roberts et al., 2014). Topic models, such those based on Latent Direchlet Allocation (Blei, $\mathrm{Ng}$, \& Jordan, 2003), can uncover meaningful patterns within collections of documents. These computer algorithms can identify syntactic patterns among texts, and these syntactic patterns often prove to have useful semantic meaning. Our methods for Structural Topic Models make three new contributions to these methods. First, STM can incorporate additional covariates about the author or context of a document. Topic models can identify patterns of responses to a course evaluation question like "How might this course be improved?" and the STM can characterize how patterns of responses to that question vary by important covariates, such a student's overall course satisfaction. Second, STM methods are built into a software package that produces a set of intuitive visualizations to support analysts in finding and exploring patterns. Finally, the software package is open source and designed for use by novices in text-analysis. These tools have opened up exciting new areas of research in many fields in the social sciences (Eggers \& Spirling, 2011; Jamal, Keohane, Romney, \& Tingley, 2014; King, Pan, \& Roberts, 2013; Stewart \& Zhukov, 2009; Stockmann, 2012; Van Atteveldt, Kleinnijenhuis, \& Ruigrok, 2008), and their development is timely as MOOCs and other large-scale online learning environments expand.

One of the primary goals of the STM project is to make these text analysis methods accessible to a wide variety of researchers and practitioners. Our focus in this paper, therefore, is not on the technical details of the STM procedure, which are described in Roberts, Stewart \& Airoldi (2014). Rather, we demonstrate how topic models may be used in the service of computer-assisted reading, leveraging computational technologies to allow educators to investigate massive quantities of student writing systematically in a reasonable amount of time. While there is no systematic research (that we are aware of) that characterizes emerging faculty practices, in our interviews with HarvardX faculty we found that instructional teams' reviews of discussion forums, assessments, and surveys are limited to the cursory, informal, and idiosyncratic. Our hope is that STM methods provide faculty with another option: to use real-time summaries of patterns found within student-produced text to make pedagogical decisions and course corrections. These same tools can also assist administrators and educational researchers in analyzing student learning in large courses.

We begin with an introduction, suitable for novices in the field, to methods of text analysis based on machine learning in order to situate the affordances of the STM. We then illustrate our approach through analyses of three different data sources drawn from MOOCs. First, we analyze responses to the aforementioned question about student rationales for signing up for an online course. Analyzing these free response items with STM allows educators and researchers to examine how students articulate their motivations in their own words, and then evaluate quantitatively how student motivations correlate with demographic characteristics or course-taking behaviour (Kizilcec \& Schneider, 2015). In 
(2015). Computer-Assisted Reading and Discovery for Student-Generated Text in Massive Open Online Courses. Journal of Learning Analytics, 2(1), 156-184.

this first example, we provide a detailed walkthrough of the workflow for using the STM package and analyzing the resulting output.

Next, we examine a second case study: the discussion forums in a humanities course from HarvardX, ChinaX Part $1 .{ }^{1}$ In many courses, especially in the humanities where large-scale, valid, reliable assessment methods are considered lacking (Ho et al., 2014), discussion forums are considered essential sites of student learning central to the objectives of the course (Reich et al., 2014a; Reich et al., 2014c). Recent research in evaluating text in these forums points to drawing small analytic samples for human coding (Stump, DeBoer, Whittinghill, \& Breslow, 2013) or using supervised learning methods to classify threads into topics (Brinton et al., 2013). We demonstrate the application of the Structural Topic Model to help instructional teams understand the range and distribution of themes in student posts, and we show how the distribution of these topics varies by important features of each document's context, such as whether or not it was "up-voted" by a peer. As a third case study, we analyze course evaluation responses from the same ChinaX course, where students were asked to write about strengths and weaknesses of the course. We demonstrate using the STM to reveal how themes in open-ended feedback can vary by other student characteristics, such as overall student satisfaction. Since these methods extend beyond MOOCs to other large-scale learning environments, we conclude with suggestions for additional applications of computer-assisted reading in other contexts.

\section{INTRODUCTION TO TEXT ANALYSIS}

The analysis of text by hand is standard practice in the social sciences, where researchers read and hand-code documents and then analyze the results. The variety of benefits to computer-assisted text analysis over hand coding include the natural improvements in speed, the ability to process high volumes of text, and the consistency of treatment of all parts of the corpus (Grimmer \& King, 2011; Hillard, Purpura, \& Wilkerson, 2008; Lowe \& Benoit, 2013). Humans often struggle with the development of complicated coding schemes (Quinn, Monroe, Colaresi, Crespin, \& Radev, 2010), and there is some experimental evidence to suggest that humans judge clusters produced by automated methods to be more semantically coherent than even an taxonomy created by the documents' authors (Grimmer \& King, 2011; Grimmer \& Stewart, 2013). The large amount of text produced in online educational environments motivates using computation to identify patterns in student-generated text. These patterns can then be presented to educators, researchers, and even students for more in-depth analysis. In this sense, the tools we discuss can be thought of as aides for "computer-assisted reading."

Automated text analysis is a form of machine learning and comes in two flavors: unsupervised and supervised. In supervised learning, the user manually labels some subset of the data, which guides the computational analysis to derive parameters for classifying the remainder of the data. Humans code a subset of documents, and computers then predict how humans would have coded the rest of the full set

${ }^{1}$ https://www.edx.org/course/harvardx/harvardx-sw12x-china-920 
(2015). Computer-Assisted Reading and Discovery for Student-Generated Text in Massive Open Online Courses. Journal of Learning Analytics, 2(1), 156-184.

of documents. In unsupervised learning, there is no user input besides the raw data, from which parameters of interest are derived. Computers find patterns in documents based on syntactic features (like the co-occurrence of certain words in the document), and humans then examine the substantive meaning of those patterns. Note that in either case, algorithms need no semantic understanding; the computer does not need to be able to understand what humans are communicating or to associate meaning with any of the words. Instead, the content and structure of corpora are sufficient to surface syntactic variations and patterns that often prove to have semantically meaningful correlates. In other words, computers can treat words as strings of arbitrary letters and find patterns in how those arbitrary strings co-occur in documents. Humans can later look at those (syntactically derived) patterns and find that they are useful and (semantically) meaningful. In our analysis, we focus on a class of unsupervised analysis called topic modelling.

\subsection{Unsupervised Topic Modelling}

Topic modelling is a particular unsupervised method that provides an approach for estimating general semantic themes within a corpus of documents (Blei, 2012). Crucially, we need not specify these themes in advance or manually annotate the input documents; the only analytic preparation required is inputting the raw textual data into a software package. Topic models use the patterns of word cooccurrences to infer semantic relationships. Loosely speaking, if two words frequently co-occur across many of the documents, we infer that they reference a similar concept or theme. The topics themselves are distributions over words. For example, consider an assignment where students write a paragraph about what they do in a typical day. One topic might be about learning, and give high probability to words such as "learning," "homework," "class," but low probability to words such as "cooking" or "eating." Each document exhibits a mixture over the topics, which encode the proportion of words within the document that the software estimates to have come from each topic. The semantic themes uncovered by the model provide a useful structure for summarizing large sets of documents. These methods complement human reading by organizing the unstructured corpus. Topic models have been widely applied throughout the social sciences and digital humanities (see Blei, 2012, and references therein).

Our focus here differs both in method and purpose with many existing applications of unsupervised learning in educational research (see Romero \& Ventura, 2007). Previous work has focused on applications towards clustering students into different learning types using their attributes, grades, and system-use statistics. When unsupervised quantitative techniques have been applied in educational contexts to text, the focus of this work has primarily been extracting data about the generation of text, engagement statistics, rather than analyzing properties of the text itself (Anaya \& Boticario, 2011; Dringus \& Ellis, 2005). Some new work in this field has moved towards models that include text features along with engagement statistics; for instance, in the MOOC context, Yang, Wen, Kumar, Xing, and Rosé (2014) use a topic model analysis of discussion forums and social network features to predict student attrition among distinct student sub-communities. We build on this this line of work with accessible 
(2015). Computer-Assisted Reading and Discovery for Student-Generated Text in Massive Open Online Courses. Journal of Learning Analytics, 2(1), 156-184.

software and methods that facilitate the substantive interpretation of topics and themes within a corpus of student writing.

\subsubsection{Comparisons to Supervised Methods}

One way to understand the uses, affordances, and limitations of unsupervised topic models is to compare them to supervised methods of text analysis. Whereas unsupervised methods uncover the most prevalent and overarching themes of the text, supervised methods can uncover 1) a category scheme dictated in advance or 2) a particular facet of the text defined in advance, such as whether the text has an introduction and conclusion or whether it should be held for review. The most common form of supervised learning is classification. In this setting, the researcher carefully reads a random sample of documents from the corpus and assigns each one to a category according to a pre-specified coding scheme. Supervised learning algorithms learn from this "training set" about how to classify the documents. They can then be used to categorize the larger set of unread documents in the corpus. Thus, the algorithm is taught how to classify through the examples in the training set, and it then extends the process to more documents than the analyst would be able to read alone. Pros and cons of these approaches have been thoughtfully enumerated in other fields of the social sciences (Bishop, 2006; Hastie, Tibshirani, \& Friedman 2009; Murphy, 2012; Grimmer \& Stewart, 2013).

One of the most well-known examples of supervised learning methods in education is automated essay scoring, which works by classifying documents along a rubric of essay quality. Then a training set of essays is scored and each essay assigned to a category by one or (ideally) multiple raters. Once these essays are scored, the algorithm classifies the remaining set of essays, perhaps flagging anomalous essays that do not appear to fit well in any category. The classifier's effectiveness can be tested by measuring the prediction accuracy within a second "testing set" of human-graded essays. Evidence from recent studies of automated essay score prediction suggests that the reliability between human graders and machine learning-based graders is similar to the reliability among human graders, at least in contexts with highly structured writing assignments that may be graded quickly (Shermis \& Hammer, 2012; Duwairi, 2006). In large-scale online environments like MOOCs, it is infeasible for faculty to evaluate the individual submissions from thousands of students, and therefore faculty who wish to assign a grade to unstructured text assignments in MOOCs need to choose among self-assessment, peer-assessment, and this kind of supervised machine learning evaluation. Even in circumstances where faculty can use supervised learning methods to assign scores to individual essays, unsupervised learning methods can uncover the themes in student writing.

Supervised algorithms are useful when researchers are interested in a particular organization of documents. These algorithms require that categories be comprehensively enumerated. In the examples that follow, we show three cases where we had no a priori expectations for categories of student writing, and we were particularly interested in topics that we and other researchers might not have considered. 
(2015). Computer-Assisted Reading and Discovery for Student-Generated Text in Massive Open Online Courses. Journal of Learning Analytics, 2(1), 156-184.

\subsection{Structural Topic Models}

One distinctive affordance of STM, compared to previous approaches to topic modelling, is the ability to incorporate additional metadata (or covariates) into the model, such as information about the author or document. This allows the analyst to "structure" the corpus prior to estimation. STM is specifically designed to leverage this existing information and facilitate accurate inferences for how the observed variables relate to the latent topics.

Running the STM provides the user with the following:

1. Estimated topics, including a small set of label words most indicative of that topic and archetypal documents from each topic

2. Relationships between covariates and topics

3. The prevalence of each topic throughout the corpus along with documents most heavily focused on each topic

4. Correlation patterns between topics (i.e., which topics are most likely to occur together within a document)

A standard workflow for the STM proceeds as follows: first, educators or researchers input a corpus of documents (discussion posts, assignments, course evaluations, etc.) into the stm package in the opensource statistical language, $R$ ( $R$ Core Team, 2012). At the same time, the user imports metadata about each document - such as the age of the author or whether a forum post was "up-voted" - into the software. These metadata covariates can then be used in the estimation of topic prevalence (how often a topic is discussed), topical content (the words used in discussing a topic), or both. The only other user input is the number of desired topics, which controls the granularity of the requested summary. Together the metadata and the number of topics define a probabilistic model that might have generated the data we observe. We then perform Bayesian inference and calculate the posterior distribution. In essence this finds the parameters most likely given both the model and the data observed. ${ }^{2}$

Once the model is fit, we can investigate relationships between the covariates and the estimated topics. For example, if we analyzed a series of open-ended responses to a question about a student's favourite aspect of a class, relationships for topic prevalence might tell us that the preferred aspects (the estimated topics) differ markedly with the overall satisfaction with the course as measured by a Likertscale item (the observed covariate). Topical content by contrast gives us insight into the words used to describe a particular topic. Thus for example, one favourite element of the class might be the

\footnotetext{
${ }^{2}$ The technical details are discussed in a companion paper (Roberts et al., 2014). Briefly, the STM is a logisticnormal mixed membership topic model. Estimation proceeds using a fast semi-collapsed, variational expectation maximization algorithm where Laplace approximations are used for the non-conjugate portions of the model. As with many modern text analysis procedures, some pre-processing is done on the texts, such as removing "stop words" (e.g., "and" and "the") and "stemming" to remove the ends of conjugated verbs and plural nouns.
} 
(2015). Computer-Assisted Reading and Discovery for Student-Generated Text in Massive Open Online Courses. Journal of Learning Analytics, 2(1), 156-184.

professor's lecture style (the estimated topic), but the words used to describe that style might differ by gender (the observed covariate).

Using covariates in the STM differs from the predictive models discussed later, such as supervised learning in that covariates might influence a topic, but the model does not force them to be connected. This helps to alleviate concerns that relationships are "baked in" to a conclusion by incorporating metadata. Rather covariates are best thought of as a way of defining subsets of the data (by age, gender, location, etc.) that may have similar patterns of topic use. In a separate work we discuss the details of the STM as well as provide simulation evidence showing its ability to uncover topic/covariate relationships (Roberts et al., 2014).

STM offers several improvements over simply performing LDA and then regressing the results on metadata of interest. First, the STM explicitly models topic correlation whereas LDA does not. Second, the STM allows for topic vocabulary - not just topic prevalence - to vary by covariates. Third, Roberts and colleagues (2014) have shown through simulations that LDA can miss important covariate relationships in the data. Finally, by explicitly including the covariate relationships in the model, we are able to include measurement uncertainty from the estimation of the latent topics into our regression analyses. In theory, as the number of documents grows extremely large, LDA will correctly recover the covariate relationships. In practice, there is evidence that even with extremely large document sets, STM yields better results both in terms of predictive power and qualitative interpretability (Roberts et al., 2014; Roberts, Stewart, \& Airoldi, 2014.)

In what follows, we provide three case studies of the use of STM for computer-assisted reading in surveys, forums, and course evaluations. The three case studies have several features in common that make them well suited for analysis with the STM. First, they all involve large corpora of texts that cannot be read in a reasonable timeframe by educators, motivating the use of computation to organize the texts. Second, they all involve domains where useful classifications of the text are not known a priori, and we can use unsupervised learning models to uncover undiscovered patterns. Finally, the three corpora have documents associated with useful metadata about the author or the document, and the inclusion of these metadata in the STM can improve the estimation of the model and reveal how themes in student writing vary across substantively interesting subgroups.

\section{COMPUTER-ASSISTED READING OF QUALITATIVE PRE-COURSE SURVEY RESPONSES WITH THE STRUCTURAL TOPIC MODEL}

We now illustrate the STM workflow and results by analyzing data about rationales for signing up for a MOOC platform. One of the largest providers of MOOCs is edX, and when students register on the edX site (a prerequisite for registering for any individual edX course) they are given a short survey including the free-response question: "Please share with us your reasons for registering with edX." While other MOOC platforms and specific courses ask students about their motivations using a variety of fixed 
(2015). Computer-Assisted Reading and Discovery for Student-Generated Text in Massive Open Online Courses. Journal of Learning Analytics, 2(1), 156-184.

response items (Breslow et al., 2013; Koller, Ng, Do, \& Chen, 2013; Wang \& Baker, 2014), this is, to our knowledge, one of the largest data sets of unstructured text where students describe their motivations for participating in online learning experiences in their own words. These data speak directly to questions about student motivation that have come to the fore as open online learning opportunities have grown dramatically (Computing Research Association, 2013). The STM allows researchers to analyze how registrants describe their motivations in their own words.

\subsection{Analytic Workflow with Structural Topic Models}

In this and the following examples, we follow five analytical steps in using the Structural Topic Model. First, we prepare the data by including the corpus of documents and relevant metadata/covariates into the software package. Second, we run the software package and produce a standard set of results and visualizations, including the list of topics in descending order of prevalence, the key words for each topic, and highly associated "archetypal" documents for each topic. Human analysis then guides the third step: we assign descriptive labels to each of the computer-generated topics by evaluating key words from each topic and archetypal documents within each topic. Fourth, we examine how topic distributions vary according to covariates of interest, to understand how important subgroups differ in their written responses. Finally, when appropriate, we propose a call to action based on the findings from the STM, which might be a pedagogical intervention or an experimental study.

In this first example, we estimate a Structural Topic Model with twelve topics examining student rationales for signing up for the edX MOOC platform. We examine the universe of all responses from edX users who by 4 August 2013 had registered for one of the first six HarvardX courses: Intro to Computer Science, Justice, The Ancient Greek Hero, Health in Numbers, Human Health and Global Environmental Change, and Copyright (Ho et al., 2014). This totals nearly a quarter million responses $(240,208)$, highlighting the need for computer-assisted reading. In the model we include several covariates: indicator variables for each course, the respondent's education level, an indicator variable for whether male, and a continuous age variable. After a small amount of automatic pre-processing, estimation of this model is done with a single line of code:

storage $<-$ stm (docs, vocab, $\mathrm{K}=12$,

prevalence $=\sim$ course+educlevel + male $+s($ age $)$, data=meta $)$

Notice the simplicity of the code syntax; one design principle of the software package is that the level of programming sophistication for conducting these analyses should be approximately equivalent to running a regression model in a typical statistical package such as R, SAS, or SPSS. In this example, we estimate 12 topics $(K)$ and the prevalence of each topic is modelled as a function of the course a person signed up for, their education level (treated as a factor variable), gender, and age (allowed to have a non-linear effect via a spline (s() function). The results are stored in an object we label "storage." A complete vignette describing all features of the stm package (Roberts, Stewart, \& Tingley, 2014), and how to use all of these features, is available at http://structuraltopicmodel.com. 
(2015). Computer-Assisted Reading and Discovery for Student-Generated Text in Massive Open Online Courses. Journal of Learning Analytics, 2(1), 156-184.

In parametric topic models such this one, it is necessary to set the number of topics. In the computer science literature it is common to set the number of topics by maximizing the predictive power of the model on a heldout sample (Wallach, Murray, Salakhutdinov, \& Mimno, 2009). However, the most predictive model may not always be the most qualitatively interpretable or substantively useful model (Chang, Gerrish, Wang, Boyd-Graber, \& Blei, 2009). Instead we see the choice of the number of topics as a decision to be made on substantive grounds that reflect the desired granularity of the summary of the corpus (Grimmer and Stewart 2013). When analyzing corpora covering a very broad array of material, higher numbers of topics may be more appropriate. We selected a K of 12 in this example, though we also estimated this model with other values of $\mathrm{K}$ for comparison, and the topics of highest interest appear in our alternate models. For those who want to use a data-driven approach, the stm package includes tools for calculating heldout likelihood as well as several other popular automated metrics (Mimno, Wallach, Talley, Leenders, \& McCallum, 2011).

\subsection{Findings from STM Analysis of Student Motivation Registrations}

Using the STM, we uncover the most common themes in student self-reported motivations for registering for edX, and in Figure 1, we present representative output from the stm $\mathrm{R}$ package.

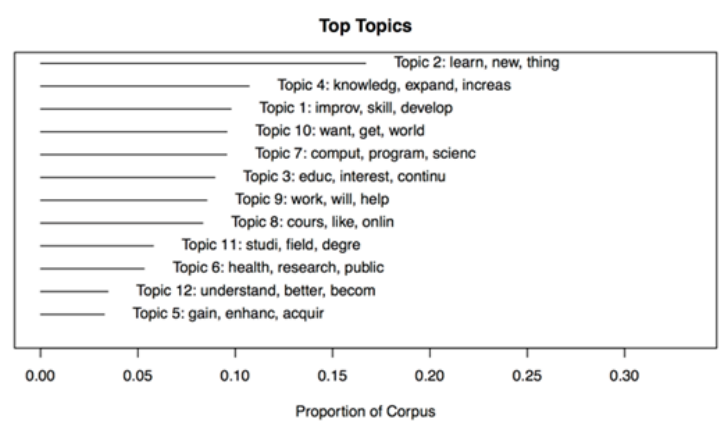

Topic 1: "Professional Development"

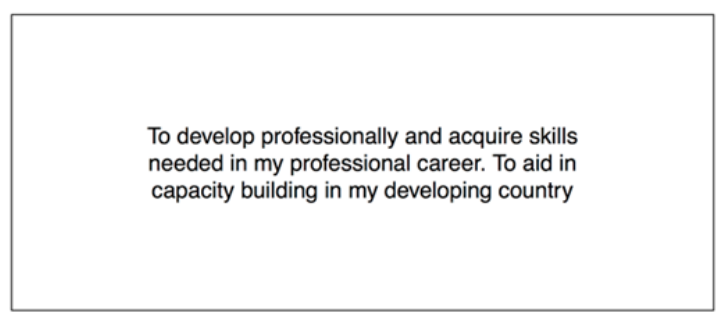

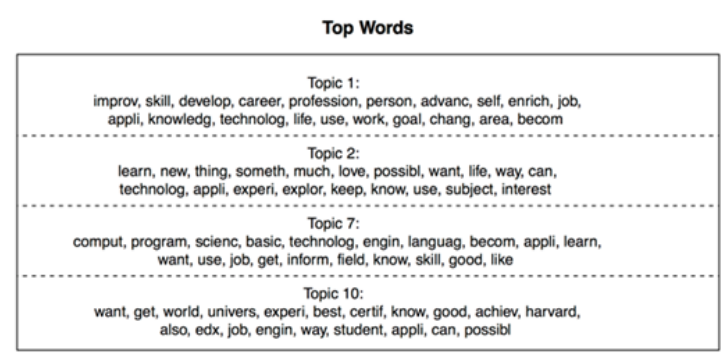

Topic 10: "Elite Association"

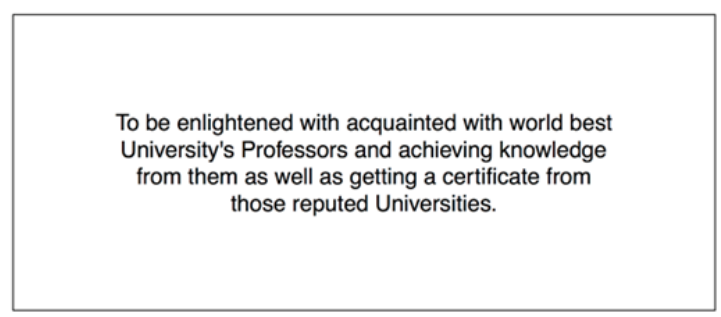

Figure 1. Representative output from a 12-topic Structural Topic Model analysis conducted in the $\mathbf{R}$ stm package from a corpus of 240,208 student responses describing their motivations for registering for edX. The top left panel shows the proportion of the corpus associated with each of the twelve topics, and three key words for each topic. The top left panel gives the twenty most probable words for four selected topics: Topic 1: "Professional Development"; Topic 2: "Lifelong Learning"; Topic 7: "Computer Science and Programming"; and Topic 10: "Elite Association." The bottom panels show highly associated texts from Topic 1 and Topic 10. 
(2015). Computer-Assisted Reading and Discovery for Student-Generated Text in Massive Open Online Courses. Journal of Learning Analytics, 2(1), 156-184.

We use two sources of data to parse the semantic meaning of these topics: 1) the word tokens mostly highly associated with each topic and 2) exemplar texts. In the top left of Figure 1, we display the 20 word stems mostly highly associated with several sample topics in their order of prevalence within the topic. In the top right panel, we show the distribution of all 12 topics across all documents. On the $x$ axis, we show the proportion of topic prevalence across all documents. For example, Topic 2 is the most prevalent in the corpus; it concerns learning new things and lifelong learning. Topic 12, which includes developing specific skills for job environments, is least prevalent. The system automatically produces the topic number and the three word tokens that most distinctly represent the topic. In the bottom left and right of Figure 1 we show for Topic 1 and Topic 10 the documents with the highest estimated proportion of topic-related words and constructs. Using these word frequency tables and exemplar texts, we then attach semantically meaningful descriptive labels for several topics: Topic 1: "Professional Development"; Topic 9: "Lifelong Learning"; Topic 7: "Computer Science and Programming"; and Topic 10: "Elite Association." The appendix provides a complete depiction of all topics (in Figure 11).
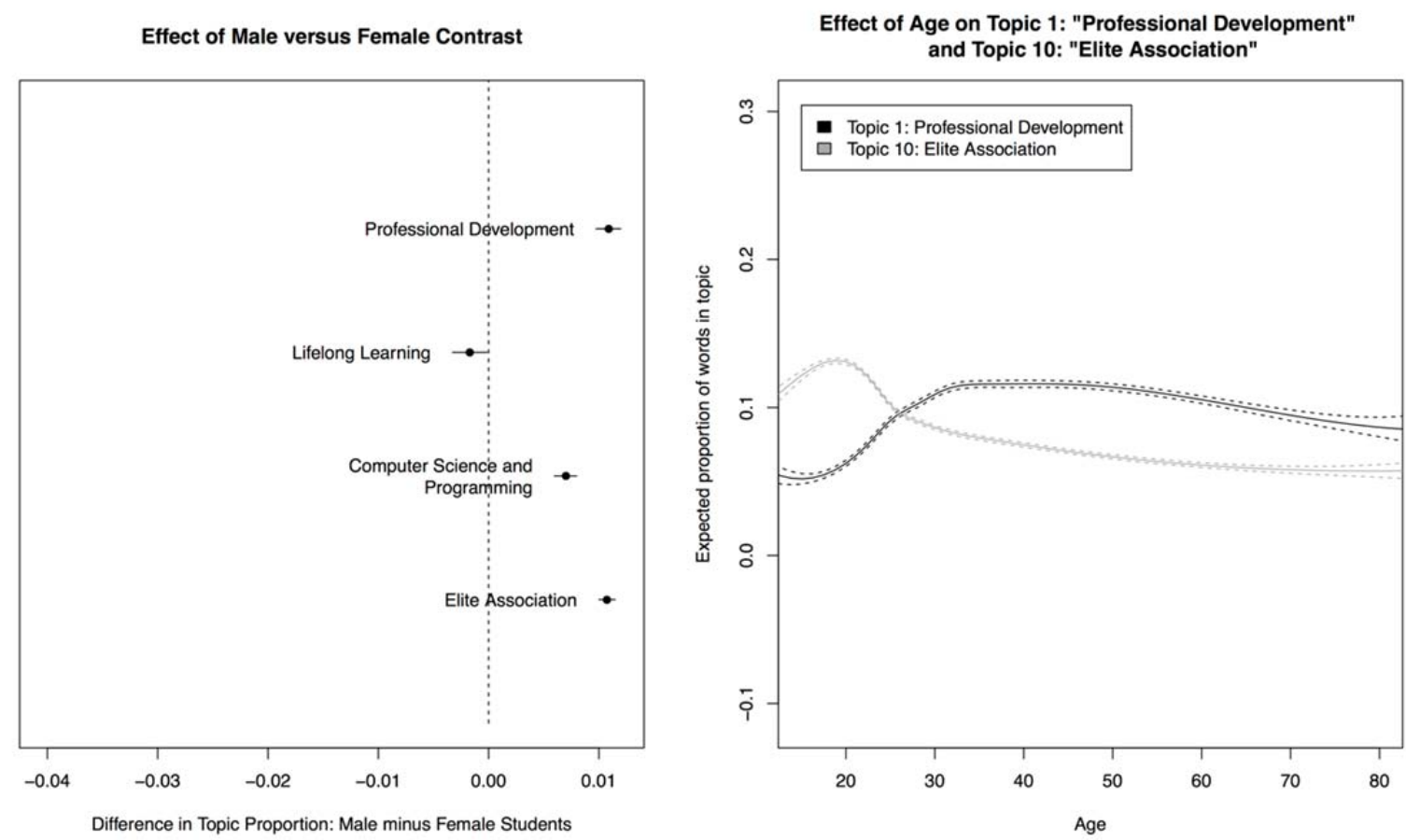

Figure 2. Results from STM analysis of a corpus of 240,000 documents describing student motivations for registering for edX. The left panel sorts four sample topics (Topic 1: "Professional Development";

Topic 2: "Computer Science and Programming"; Topic 9: "Lifelong Learning"; and Topic 10: "Elite Association") by their respective use by males relative to females. Positive numbers indicate that males were more likely to write about the topic. The right panel shows the effect of age on usage of

two sample topics. For example, younger MOOC registrants appear more motivated by elite association than career development, whereas older MOOC registrants are more likely to write about career development. 
(2015). Computer-Assisted Reading and Discovery for Student-Generated Text in Massive Open Online Courses. Journal of Learning Analytics, 2(1), 156-184.

The topic model reveals both predictable and surprising student motivations. Topic 1, for instance, uncovers students who describe their motivations as instrumental and professional in nature; they register for MOOCs to advance their careers. Given the practical nature of several of the early HarvardX courses, like Health in Numbers (biostatistics and epidemiology) and Computer Science, this topic is to be expected. Topic 10 shows the importance of associating with a leading university. This is one of the most commonly expressed reasons for wanting to sign up for a MOOC, and echoes the edX marketing language that the platform offers "the best courses, from the best professors, from the best universities." This suggests that this element of elite branding is front-of-mind for many participants when signing up for edX. While in retrospect the importance of this topic makes sense, this dimension of elite affinity does not always appear in other surveys of student motivations in the MOOC literature. The topic model here uncovers an important dimension of student motivation that is presently underresearched. These findings informed the design of the 2013-2014 HarvardX and MITx pre-course surveys, which now ask students about the importance of career advancement and elite affiliation in their registration decisions.

After examining the topics themselves, we go on to examine how these motivations differ across substantively interesting sub-populations, which we can do with both dichotomous and continuous measures. In Figure 2, we show how the prevalence of topics differs by gender and age. In the left panel, we plot the difference in the expected proportion of words within the topic for men minus the expected proportion of words within the topic for women. When calculating effect, the values of all other variables are set at their sample median values. ${ }^{3}$ The lines give $95 \%$ confidence intervals on the difference including measurement uncertainty. Positive numbers indicate that males were more likely to write about the topic. For instance, Topic 10 describes the desire to acquire computer science knowledge, and it was the topic most heavily correlated with the respondent being male. We would predict that documents produced by men would have more words and constructs related to computer science than documents produced by women; on average, the proportion of words in a document from this topic will be .006 more for men. While small, given our large sample size, this is statistically significant. Computer science MOOCs disproportionately enrol men - the student body of the fall 2012 HarvardX Introduction to Computer Science course was $79 \%$ men - and this unsurprising result gives us greater confidence in the performance of the model. The left-hand plots can be produced with two lines of code, the first to calculate the necessary quantities, and the second to produce a plot:

\section{prep <-estimateEffect $(c(1,2,7,10) \sim$ course+educlevel+male+s(age), storage, meta=meta)}

\section{plot.estimateEffect(prep, "male", model="z", method="difference",}

\footnotetext{
${ }^{3}$ We note that the STM model supports a broad array of specifications, including interactions between variables and allowing for non-linear effects through the use of splines. Here we do not include interactions but we do allow the effect of age to be non-linear.
} 
(2015). Computer-Assisted Reading and Discovery for Student-Generated Text in Massive Open Online Courses. Journal of Learning Analytics, 2(1), 156-184.

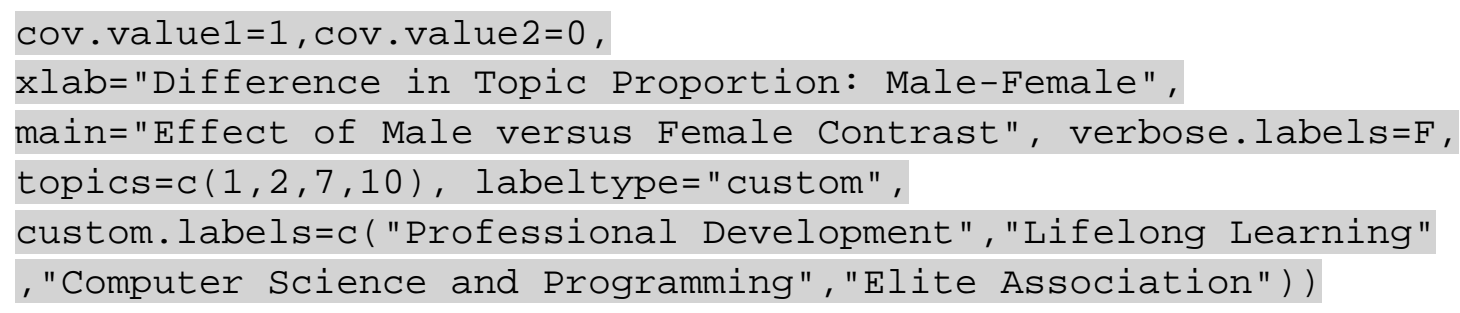

Next, we show how to analyze the influence of a continuous covariate: the age of a student. To analyze a continuous covariate, we plot the predicted proportion of a document that comprises a topic as a function of age. To do this requires a single line of code:

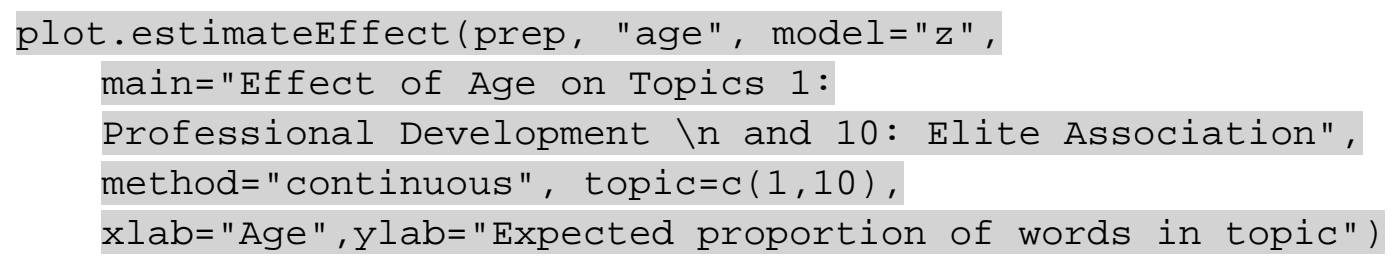

In the right-hand panel of Figure 2, we show the effect of age on two topics in the corpus, Topic 1: "Professional Development" and Topic 10: "Elite Association." On the X-axis we show age, and on the $Y$ axis, we show the expected proportion of words in respondents' text that come from a particular topic. The dashed lines provide $95 \%$ confidence intervals. The importance of allowing a non-linear relationship with age is quite apparent. Among this cross-sectional cohort, younger MOOC registrants appear more motivated by elite association than career development, whereas older MOOC registrants are more likely to write about career development. ${ }^{4}$ These findings could inform recruitment efforts by universities and MOOC providers, and they suggest possibilities for segmenting marketing, where students of different ages receive recruitment materials highlighting different themes. These findings could also inform the design of more personalized learning environments; for example, by providing older students with more examples from industrial or commercial contexts.

To review, we analyzed nearly a quarter-million statements about why an individual was signing up to take a MOOC, and we used the STM to identify a set of syntactically related topics that represented substantively interesting response patterns. Evaluating all quarter-million documents would be infeasible without computer assistance, and even hand-coding methods could require coding a random sample of thousands of documents to uncover more rare topics. We then show how these distributions vary by age and gender; the STM is unique in its utilization of covariate information in this way and as such holds promise for use across educational data.

There are two caveats to using this method. First, the method organizes data for human analysis, uncovering patterns across large amounts of text, but ultimately the utility of these clusters depends

\footnotetext{
${ }^{4}$ We retained and used in estimation cases where individuals list low or very high ages. Results in these regions should probably be taken cautiously. Our ability to use a spline function means they are not influential outliers.
} 
(2015). Computer-Assisted Reading and Discovery for Student-Generated Text in Massive Open Online Courses. Journal of Learning Analytics, 2(1), 156-184.

upon thoughtful and necessarily subjective assignment of meaning to these clusters. Second, unsupervised topic modelling can uncover clusters of interest, but it cannot assign documents to a predefined taxonomy or assess how the distribution of documents fits into an ideal distribution. Supervised methods are more appropriate for these purposes.

\section{ADDITIONAL EXAMPLES OF STRUCTURAL TOPIC MODELLING IN MASSIVE OPEN ONLINE COURSES}

In this section, we examine the application of the STM to student discussion forums posts and course evaluations. Both examples come from a HarvardX course, ChinaX, ${ }^{5}$ conducted on the edX platform in the fall of 2013. When finished, ChinaX will span nearly 15 months of course content offered in ten parts, with separate final grades and certificates for each part. The course was taught by Professors Peter Bol and William Kirby, and Part I explored the political and intellectual foundations of China. Part I launched on 31 October 2013 and finished on 23 December 2013 with 33,479 students registering for the course and over 2,000 students earning a certificate of completion. As with many online courses in the humanities, contributions to the forums are required to earn a certificate. Students are also asked to complete course evaluations and to provide open-ended feedback that can help the instructional team to make improvements in the course. We demonstrate how the STM helped the ChinaX instructional team see the broad themes in discussion forums and survey feedback and begin to use that feedback to iteratively improve subsequent sections of the course.

In each example, we continue to provide graphs of various key quantities from the STM model. To review: topic words give information about the top words used in each topic, listed in order of their weight in that topic, to understand the general language of a topic. Highly associated texts are specific examples of documents characteristic of a particular topic. In combination with the top words in each topic, they allow researchers to understand specific instances of topic usage, supplementing the overall analysis with example documents. Covariate relationships describe the relationship between topic usage and covariates values. Topic prevalence gives the relative usage of the topic across the corpus. This allows the user to find the overall themes of the corpus with relative weight of the different themes in the discussion. All analysis is done using the free, open-source R package stm that features a rich set of functions requiring very minimal programming knowledge of the user, equivalent to an understand of basic data entry and statistical testing in SPSS, SAS, or Stata.

\subsection{Discussion Forums}

Discussion forums in MOOCS provide an opportunity for students to develop and demonstrate their understanding, to ask questions, and to interact with each other and with course teaching staff. Literature on the use of forum-based learning in online education indicates that online forums can be

\footnotetext{
${ }^{5}$ https://www.edx.org/course/harvardx/harvardx-sw12x-china-920
} 
(2015). Computer-Assisted Reading and Discovery for Student-Generated Text in Massive Open Online Courses. Journal of Learning Analytics, 2(1), 156-184.

important for their ability to facilitate debate, networking, and interaction with instructional staff (Mak, Williams, \& Mackness, 2010). Furthermore, online discussion forums can increase individual participation and in the best instances promote a collaborative learning environment that allows for high-level critical thinking (Thomas, 2002). The best online discussion forums are facilitated by instructional staff, but in MOOCs and other large-scale learning environments, students can generate text at a pace that far exceeds an instructional team's capacity to read.

One solution to these problems has been the use of voting to promote comments of importance to the attention of students and the instructor. By design, these comments promote a biased representation of the course, where popular comments become more visible. These comments can present a skewed version of the course to the instructors, often times drawing their attention to the writings of first posters or most enthusiastic contributors rather than the writings of more typical students. In these circumstances, the use of computer-assisted reading can enable the teaching staff of a MOOC or other online-discussion-intensive course to review the forums and gain an overall understanding of the discussion topics and specific, representative points (Dringus \& Ellis, 2005). The STM provides exactly this functionality. The inclusion of covariates within a topic model allows data such as up-votes to factor into a model. The STM model allows the instructor to observe general trends in discussion topics, view particular posts that capture the essence of the topics, and understand the correlation between topic usage and votes within the forum. This lets the instructor observe what kinds of conversations are provoked by the instructional content of a lesson or unit.
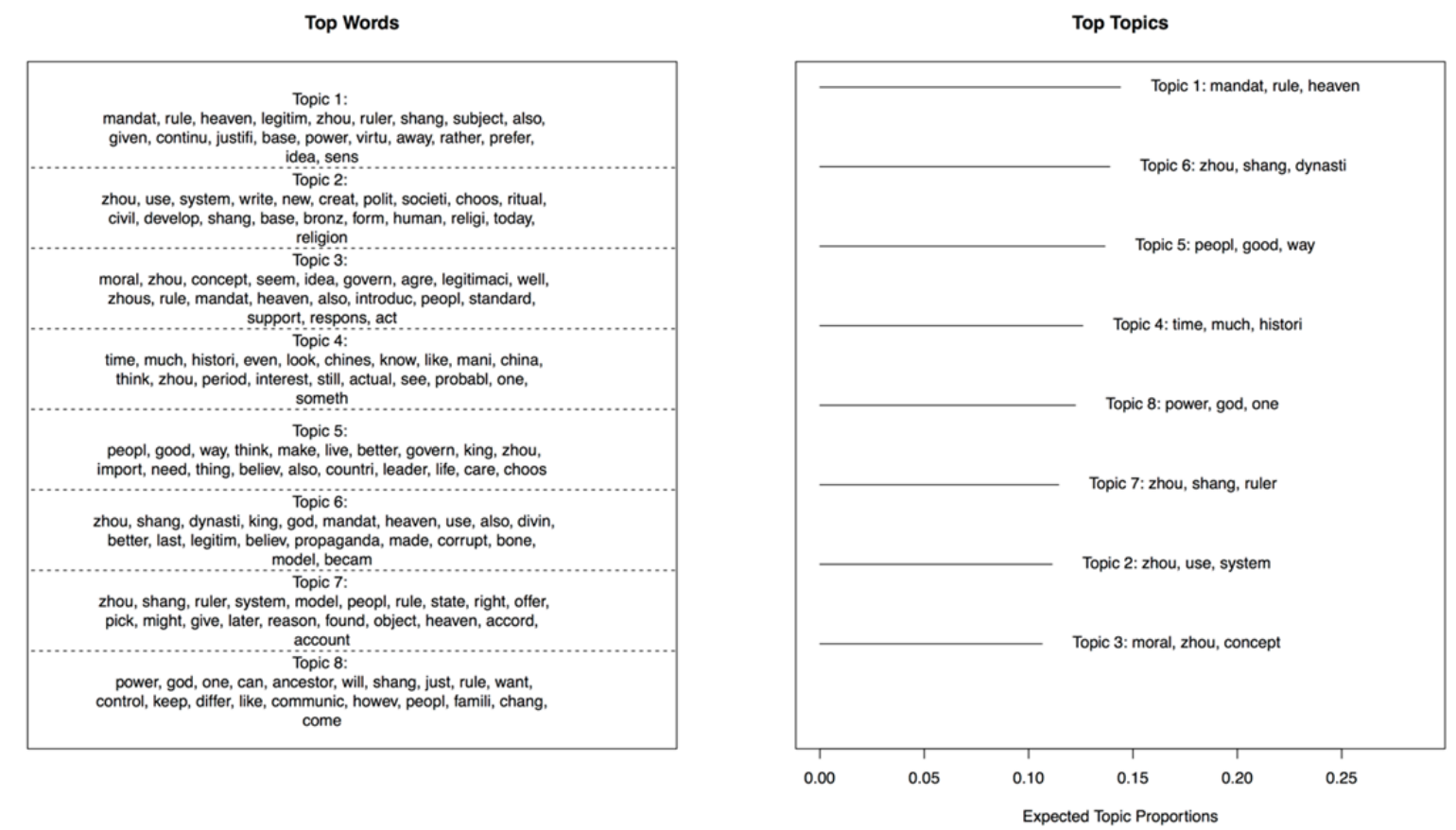

Figure 3. Results from an 8-topic STM analysis of a corpus of discussion forum posts from ChinaX examining the Zhou and Shang dynasties in China. The left panel shows key words associated with the eight topics, and the right panel shows the distribution of topics. 
(2015). Computer-Assisted Reading and Discovery for Student-Generated Text in Massive Open Online Courses. Journal of Learning Analytics, 2(1), 156-184.

In the following analysis, we explore text data from a pair of discussion threads, each with more than 1,600 posts, from Part I of ChinaX. The course explores multiple dimensions of ancient Chinese history and culture, but the reader need not have a deep background in Chinese history to begin to understand our analyses. The STM provides semantically coherent topics with example posts related to each topic, which allows a non-specialist to begin understand the discussion themes. In modelling these two corpora of texts, we also include as a covariate whether or not each post was up-voted. Figure 3 is the general result of the STM modelling forum posts where students were asked to compare the Zhou and Shang dynasties for governing style: "Although both Shang and Zhou are at the beginning of China's history, later dynasties would look to Zhou as the model of civilization, rather than Shang. What did Zhou offer that Shang did not? What did Shang have that later people might have objected to?" As in the previous example, in Figure 3, the top left panel presents the top words in each topic, while the top right shows their respective prevalence throughout the corpus.

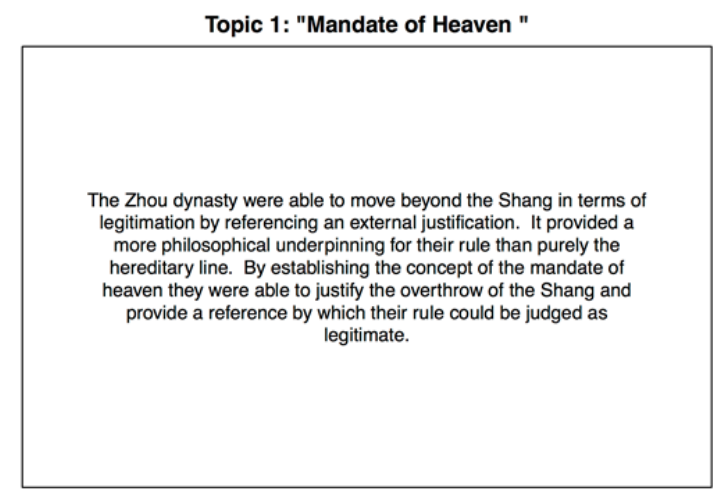

Topic 5: "Populist Governance"

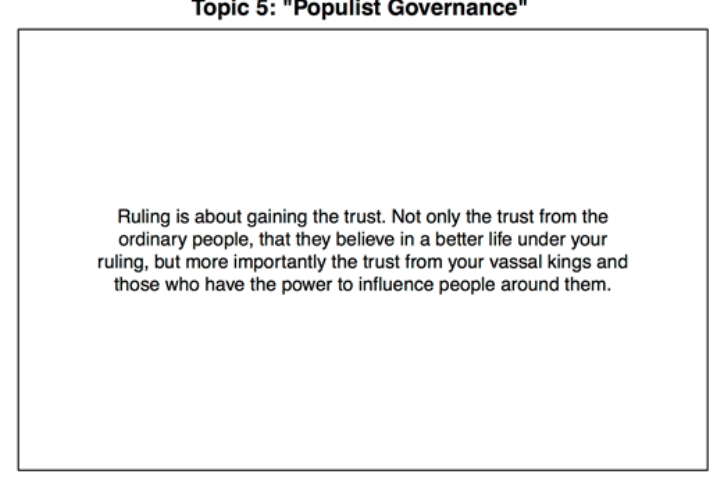

Topic 2: "Innovations in Statecraft"

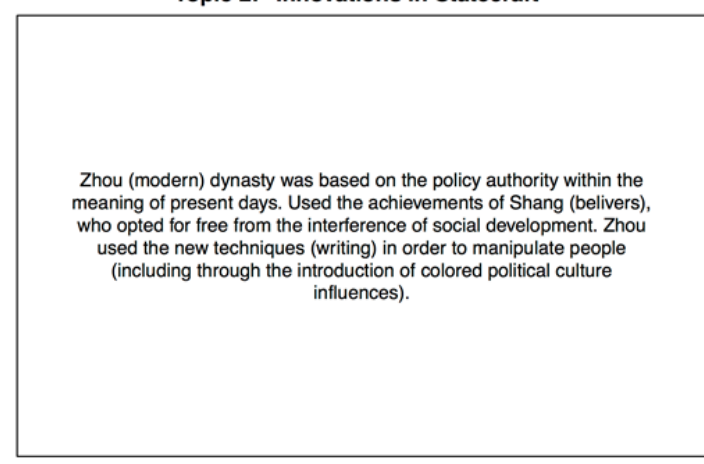

Topic 8: "Religious Power"

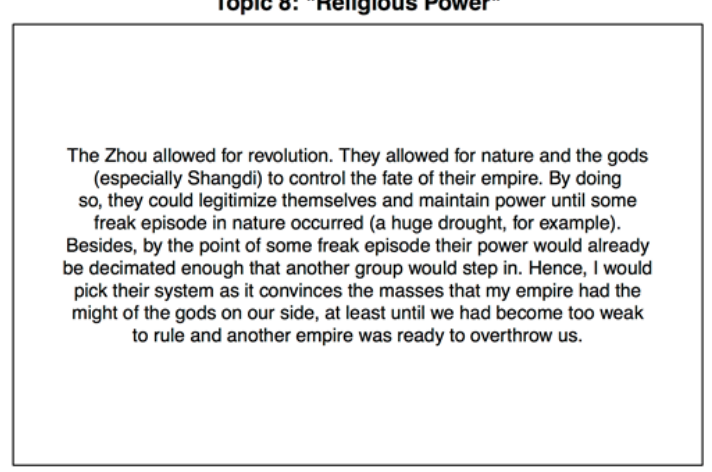

Figure 4. Example forum posts on Shang and Zhou for Topic 1: "Mandate of Heaven"; Topic 2: "Innovations in Statecraft"; Topic 5: "Populist Governance"; and Topic 8: “Religious Power."

Appropriately, the most prevalent topics in the corpus have to do with issues of religious and popular legitimacy. One of the main ideological innovations of the Zhou dynasty was the notion of the "Mandate of Heaven": that rulers maintain their position by the grace of the gods, and dishonorable rulers can have their mandate revoked (as "occurred" when the Zhou violently overthrew the Shang). Virtuous 
(2015). Computer-Assisted Reading and Discovery for Student-Generated Text in Massive Open Online Courses. Journal of Learning Analytics, 2(1), 156-184.

rulers maintain the support of their people and earn the favour of the gods. These ideas are critical to the art and writing of the Zhou period, and Topics 1, 6, 5, and 8 are clearly related to these key issues.

When comparing topics, the stm package can produce visual depictions of the difference between topical themes, as shown in Figure 5, to see overlap as well as subtle differences in similar topics. Here we have compared Topics 5 and 8, to show some of the differences in language between discussions of religious power ("Religious Power") from those of populist issues ("Populist Governance").

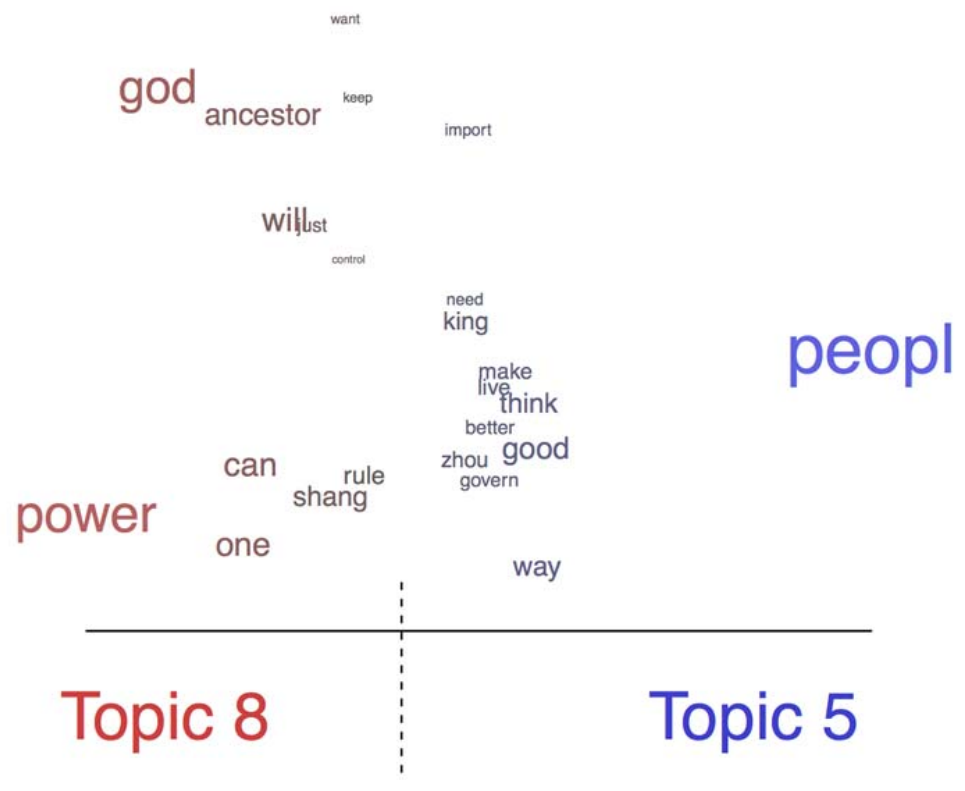

Figure 5. Contrast in words between Topic 8: "Populist Governance" and Topic 5: "Religious Power." Words further to the outside are more heavily associated with a single topic. Larger words have a greater weight within that topic. The dashed line represents the split between words that have greater weight on Topic 8 versus Topic 5 .

While these intellectual advances are important to understanding the period, the content in this unit of ChinaX also explored how these new ideologies required innovations in statecraft to spread. During this period, rulers found new uses for writing, bronze vessels, religious rituals, and public speeches to earn and maintain legitimacy in the eyes of the people. For instance, many religions functions that had been relatively apolitical in the Shang became more politicized in the Zhou. Only one topic, and one of the least prevalent topics, coheres with these ideas about advances in statecraft: Topic 2, which included words like "system," "write," "new," and "bronze."

The STM model shows that across the hundreds of posts and comments in the thread, students wrote more about the ideas that the Zhou developed to assert their legitimacy rather than the emerging statecraft methods they used to spread these ideas. This provides the instructional team with a qualitative reflection on their teaching that would be difficult to obtain without reading a substantial sample of the discussion forum. If both the ideas and the statecraft are critical to their interpretation of 
(2015). Computer-Assisted Reading and Discovery for Student-Generated Text in Massive Open Online Courses. Journal of Learning Analytics, 2(1), 156-184.

this period of history, then these analyses suggest that in a revision of the course content, the instructional team might consider revising the unit to place more emphasis on these ideas that students wrote less about. In this MOOC context, the STM gives faculty rapid feedback on how students are reacting and responding to instructional materials.

The forum analyses can be enhanced by including covariates, such as whether a post was up-voted by a peer in the forums. Figure 6 summarizes a set of posts in which students were asked to compare principal thinkers across Chinese history from Confucius through the era of the "One Hundred Schools." The top left graph gives the top words in several topics that we discuss below, while the top right graph gives us the respective topic weights in the corpus. In Figure 7, we show specific examples characteristic of four topics discussed below. As with the previous example, we can use these topics and word lists to review how students interpreted course materials. For instance, we are pleased to find that students discuss Han Fei and associate him with the law, as Han Fei's development of Legalist theory is an important intellectual advancement from the era. We also see that many students write responses that include comparisons of multiple thinkers. Topic 2, for instance, includes comparisons of Laozi and Mencius. Interestingly, the most prevalent topic, Topic 4: "Vague Language," includes no word tokens referencing any specific philosophers or philosophies. These distributions tell us what students wrote about; next we turn to an examination of which topics were most likely to be part of a post that received an up-vote, which provides one measure of how other students responded to these posts.

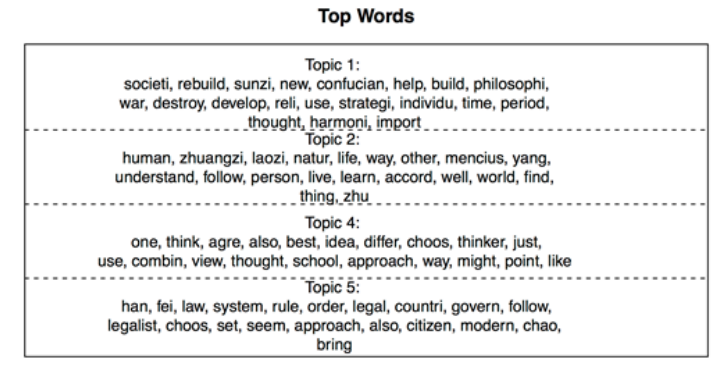

Effect of Pro-Vote

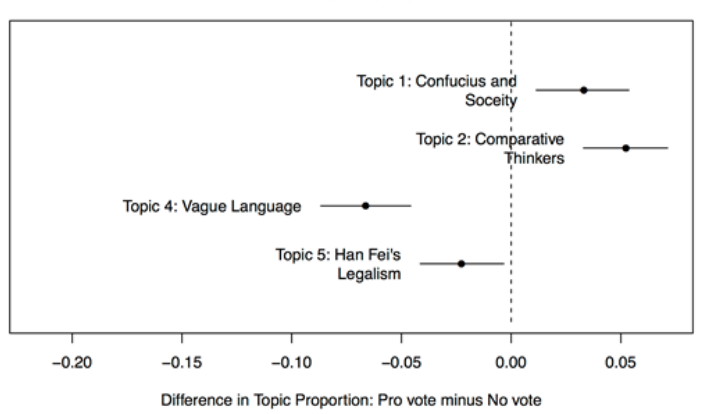

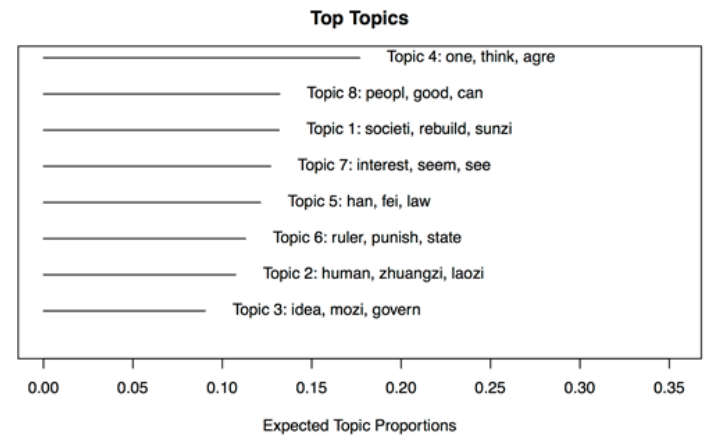

Expected Topic Proportions

Figure 6. Output from the STM analysis of a discussion forum with 1,715 posts concerning ancient Chinese philosophers. Words associated with Topic 1: "Confucius and Society"; Topic 2: "Comparative Thinkers"; Topic 4 "Vague Language"; and Topic 5: "Han Fei's Legalism" appear in the top left panel. The top right panel shows the frequency distribution of topics the across the corpus. The bottom left panel shows the effect of topic usage on receiving at least one up-vote. 
(2015). Computer-Assisted Reading and Discovery for Student-Generated Text in Massive Open Online Courses. Journal of Learning Analytics, 2(1), 156-184.

Topic 2: "Comparative Thinkers"

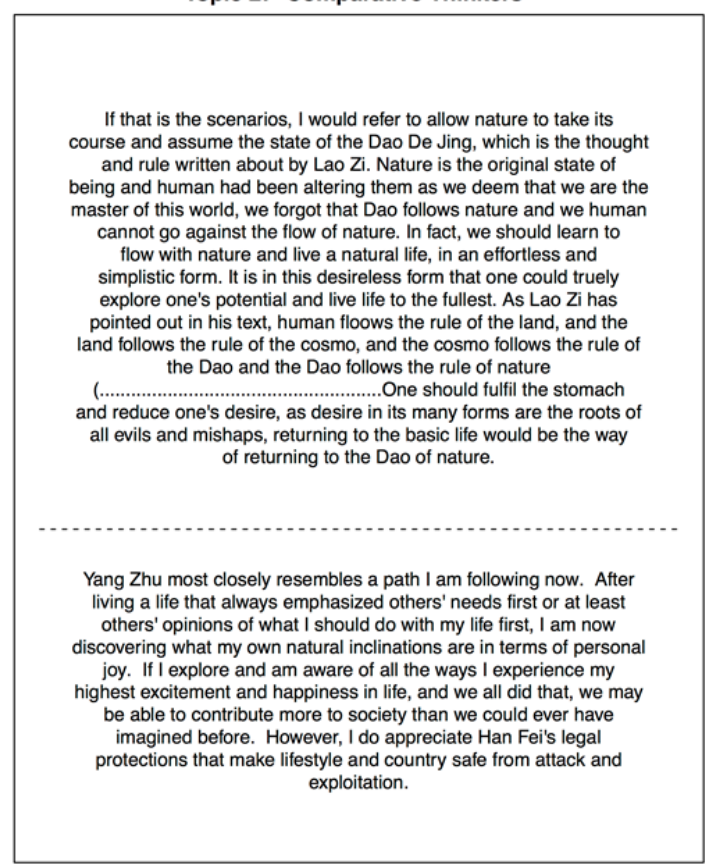

Topic 5: "Han Fei's Legalism"

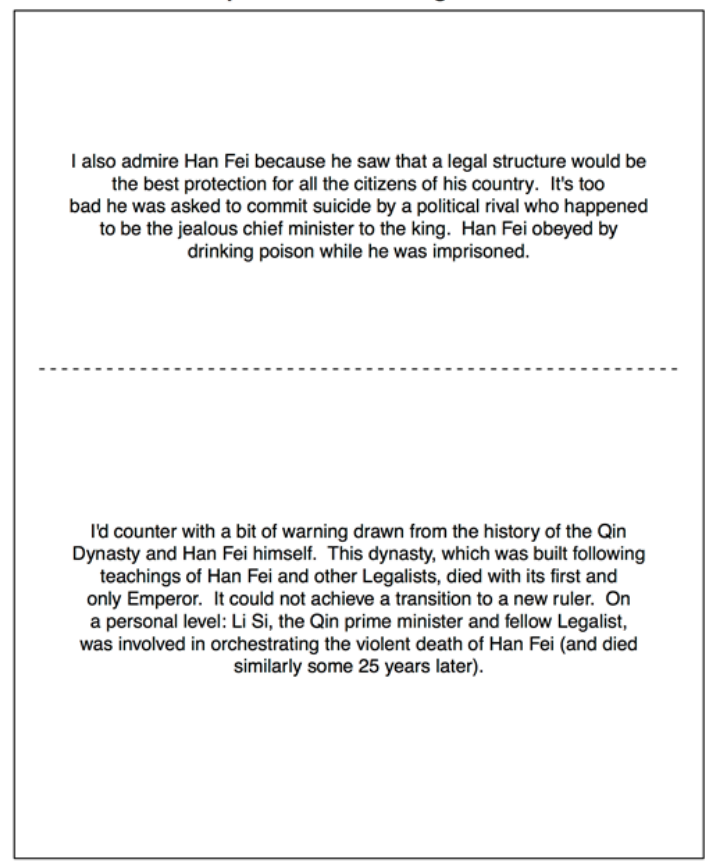

Topic 4: "Vague Language"

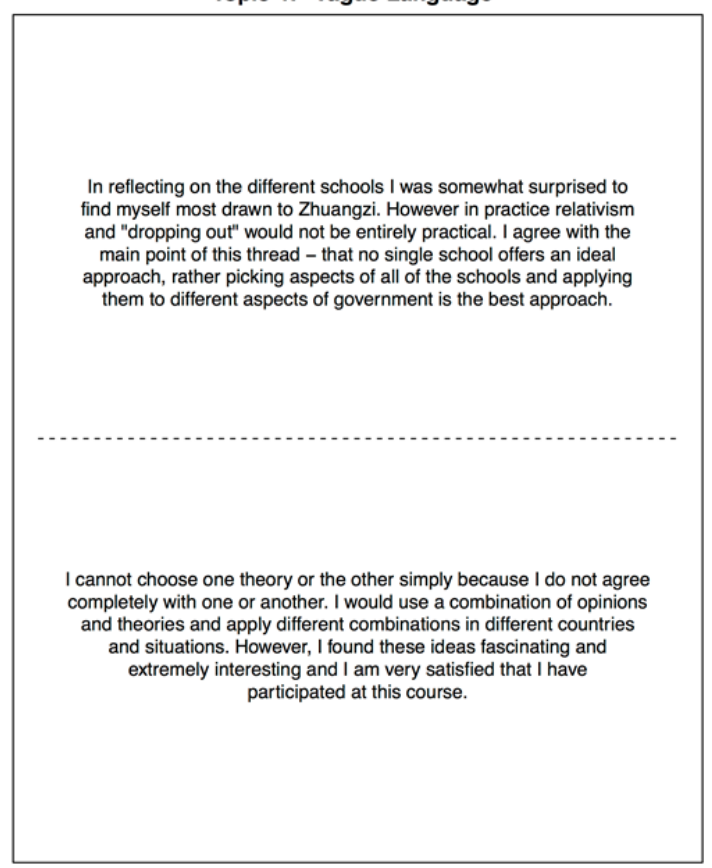

Figure 7. Example forum posts from ChinaX comparing ancient Chinese Philosophers from four topics: Topic 1: "Confucius and Society"; Topic 2: "Comparative Thinkers"; Topic 4: "Vague Language"; and Topic 5: "Han Fei's Legalism." 
(2015). Computer-Assisted Reading and Discovery for Student-Generated Text in Massive Open Online Courses. Journal of Learning Analytics, 2(1), 156-184.

The bottom left panel of Figure 6 shows us the relationship between topic usage and receiving at least one up-vote in the forums. Notice that Topic 2 ("Comparative Thinkers") is most associated with having at least one up-vote, and in contrast, Topic 4 ("Vague Language") was most negatively associated with having an up-vote; students did not up-vote comments that reflected indecisiveness or a lack of specific evidence. Interestingly Topic 5 ("Han Fei and Legalism") was also less likely to be up-voted. In a deeper analysis of these posts, it would be worth exploring whether these posts were less likely to be up-voted because they were poorly written or had some problem in argumentation, or if Han Fei's Legalism simply proved unpopular with a modern audience.

This analysis motivates several kinds of experiments in sharing these findings directly with students as a way of offering feedback on participation in the discussion forums with concrete examples. For instance, instructors could show students the evidence that vague responses received few up-votes while responses with specific ideas and evidence received more, and then show exemplars of each. This kind of feedback on the specific characteristics of high-quality responses in a forum would hopefully help students write better responses. Ideally, faculty would test these ideas by showing the feedback to a random subsample of students to determine if this kind of feedback helped some improve their writing. We could also show students the evidence of their own leanings and biases, for instance by demonstrating the community's low regard for Han Fei. We believe this kind of computer-assisted reading and real-time display of textual data could play an important role in providing students feedback on discussion forums too large for rapid human analysis.

In just a few graphs, the STM offers a thematic overview of the student responses from two entire forums worth of data. We have the ability to see the topics by frequency and word choice and to dive into those topics by looking at archetypal posts. This allows us to monitor student understanding of topics to prevent gaps in knowledge or misunderstanding while also understanding the discussion in terms of broad themes. These results can easily be incorporated into later coursework or when retrospectively evaluating the success of a course. At present, one of the most challenging aspects of incorporating forums in MOOCs is that they rapidly become far too extensive for any student or faculty member to follow, and the STM offers a toolset for finding patterns amid these wide-ranging conversations.

\subsection{Class Feedback}

Research suggests that faculty who reflectively incorporate feedback from student evaluations improve their teaching, as measured by subsequent evaluations (Winchester \& Winchester, 2014). In small-scale teaching environments, it is possible to read and analyze an entire set of student evaluations that include qualitative feedback. But as class sizes grow, especially in large-scale online learning environments, reading thousands of open-ended student responses becomes logistically infeasible. We present a strategy that allows faculty to ask for rich qualitative feedback from many thousands of students and then use computer-assisted reading methods to find patterns of feedback and to uncover typical suggestions or concerns. 
(2015). Computer-Assisted Reading and Discovery for Student-Generated Text in Massive Open Online Courses. Journal of Learning Analytics, 2(1), 156-184.

At the close of the first eight-week mini-course of ChinaX, participants were invited to complete a course evaluation, which combined both open- and fixed-response answers. We have responses from 1,057 students for which we have complete covariate information. This represents approximately $2.8 \%$ of all registrants of the course and $42.3 \%$ of all who explored over half of the units of the course. Students who completed the survey were overwhelmingly those who persisted throughout the entire course; $79.9 \%$ of survey respondents earned a certificate in the course compared to $5.4 \%$ of all registrants. Our findings here describe how a subset of successful students evaluated the course.

Students were asked to articulate their feedback on the course in two open response questions:

- What were your favourite aspects of this mini-course so far?

- What could the ChinaX team do to improve your learning experience?

Students were asked additional fixed responses questions such as "Overall, how satisfied were you with this mini-course?" with response anchors ranging from "Very Dissatisfied" to "Very Satisfied." In analyzing and triangulating responses from these questions, we can provide a nuanced picture of how substantively interesting subgroups evaluate the course.

Figure 8 shows the results from a seven-topic STM of student responses to their favourite aspects of the course for several selected topics. ${ }^{6}$ The most prevalent topic (Topic 3) connects to the relationship with the course faculty and guest lectures and specifically references "office hours." The ChinaX instructional team and faculty created video "office hours" every other week during the course, which were filmed as the course progressed, in contrast to the rest of the content, which was prepared well in advance. In these office hours, the course faculty provided a response to the discussion forums, highlighted ideas from the previous week, and showcased material to come. It was a time-intensive addition to the course, and the ChinaX teaching staff was gratified to see that students responded positively to the efforts. These findings help persuade the instructional team to continue investing effort into producing the office hours videos in subsequent weeks of the course. More broadly, the prevalence of the topic suggests that rapport with faculty is important even in these distance courses (Murphy \& RodriguezManzanares, 2012). The second most prevalent topic was about the course content, Topic 2, and includes specific reference to the short videos, most no longer than five minutes. This student feedback lends some evidence to the assertion that shorter videos may be a particularly appropriate content medium for the MOOC context (Guo, Kim, \& Rubin, 2014). ${ }^{7}$

\footnotetext{
${ }^{6}$ In this STM, we include as topic prevalence covariates our satisfaction measure, levels of familiarity with the subject matter, reasons for taking the course, age, and gender.

${ }^{7}$ Other topics dealt with more substantive topics like Chinese culture and history, and more general comments about enjoying the class.
} 
(2015). Computer-Assisted Reading and Discovery for Student-Generated Text in Massive Open Online Courses. Journal of Learning Analytics, 2(1), 156-184.

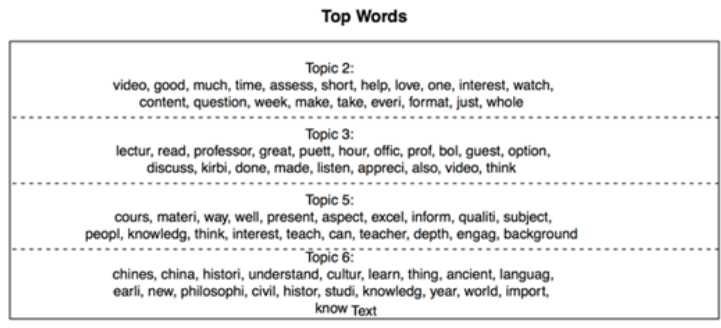

Topic 2: "Video Material"

The sets of short videos that make up the main content are a really good format ... a big improvement over single long lectures. The mak it much easier to give full concentration to all of the content, and more convenient to manage in my spare time.

I like the short videos. Often I just have a few minutes at the same time and the short videos are good for this reason.

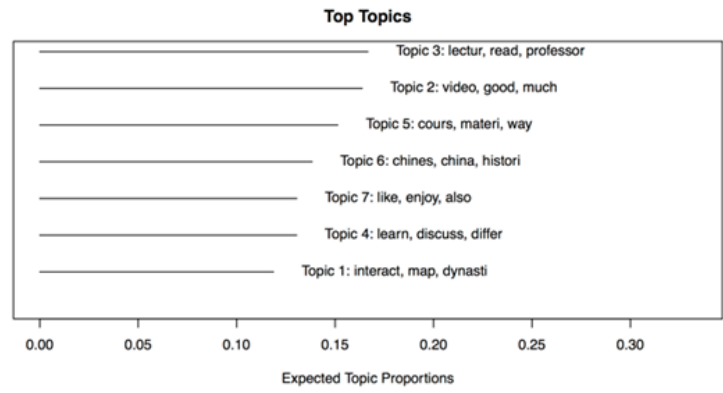

Topic 3: "Professors and Lectures"

The lectures, office hour, Prof. Puett's lectures, and mini Course conclusion especially.

The office hour is very good. It made me feel as if we were so close. Thanks to the team and especially professor Peter Bol and professor Bill Kirby. I do enjoy your lecture. I

Figure 8. Output from 7 topic STM analysis of 1,057 responses to ChinaX course evaluation. The top left panel includes top words from four topics, Topic 2: "Video Material"; Topic 3: "Professors and Lectures"; Topic 5: "Course Materials"; and Topic 6: "Chinese History in Context." The top right panel includes the proportion of all seven topics across the corpus. The bottom panels have highly associated texts from Topics 2 and 3.

Figures 9 and 10 show the results of a ten-topic STM on the responses regarding what elements of the course could be improved. Here we focus our analysis on the influence of different levels of student satisfaction. Nearly all students who take the survey were either "Very" or "Extremely" satisfied with the course, so we are examining a narrow range of happy, successful students. Nonetheless, the differences in topic prevalence by student satisfaction are revealing. We see that Topic 4 ("Assessments") was associated with less satisfied students, and from the listing of the top words associated with this we recognize that these students took issue with the assessment and question format of the course. Our text samples indicate that these students felt that the questions were ambiguous or needlessly tricky. By contrast, more satisfied students raised issues with the discussion forum platform used by edX, Topic 2 . These students complained about technical issues that prevented them from easily engaging with other students; they wanted to be able to participate even more fully!

Topic 7 includes references to Chinese characters, and it was the second most prevalent topic. While the course was ongoing, the ChinaX course team received several emails asking that more Chinese and English characters be displayed in the videos. The emails indicated that students were using the course to learn English or Chinese. These emails were sporadic and idiosyncratic, so the team debated whether to devote additional resources to adding Chinese characters to the videos. The prevalence of this topic in the course evaluation provided additional evidence that this was a widespread interest, and the course team decided to invest more resources in displaying more Chinese and English text in the videos 
(2015). Computer-Assisted Reading and Discovery for Student-Generated Text in Massive Open Online Courses. Journal of Learning Analytics, 2(1), 156-184.

themselves. The computer-assisted reading of these course evaluations helped faculty to confirm the importance of this issue for learners and to improve this dimension of the course iteratively as it progressed.

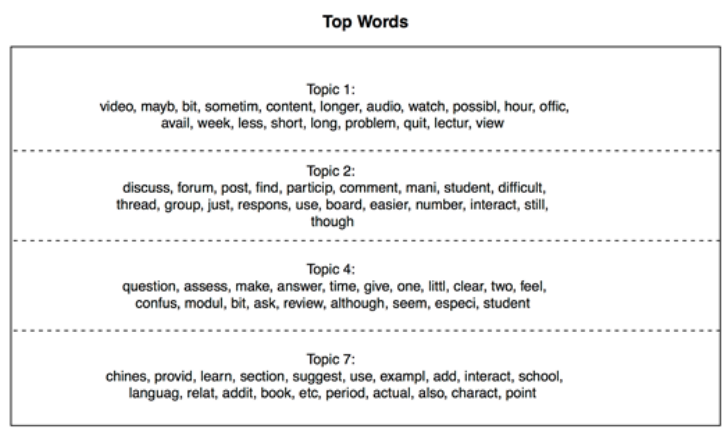

Effect of Satisfaction on Topic Usage

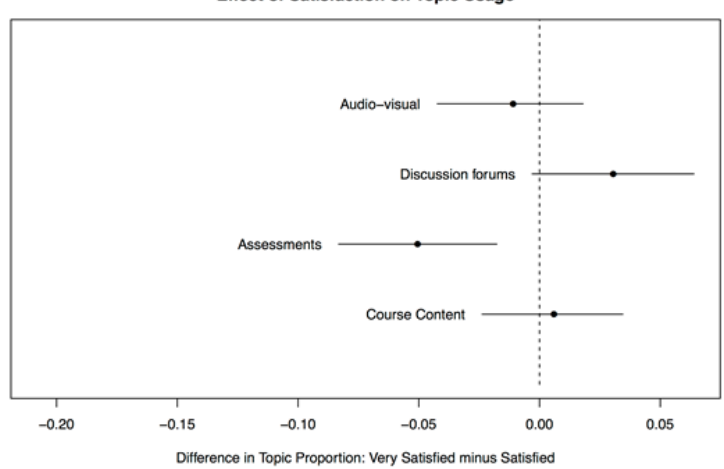

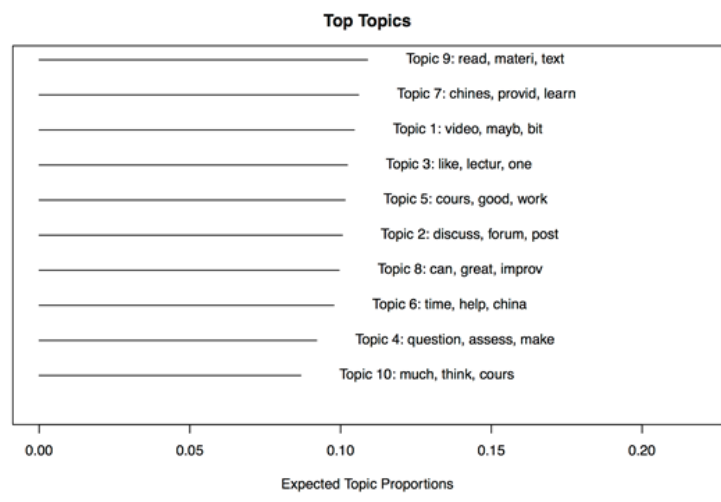

Expected Topic Proportions

Figure 9. Output from 10-topic STM model examining 1,057 course evaluations concerning what could be improved in ChinaX. The top left panel includes top words from four sample topics: Topic 1:

"Audio-visual"; Topic 2: "Discussion forums"; Topic 4: "Assessments"; and Topic 7: "Course Content." The top right panel shows the proportion of each topic across the corpus. The bottom left panel shows the effect of topic usage on being "Very Satisfied" versus "Extremely Satisfied."

In each of these examples, the STM successfully modelled responses to course evaluations, providing useful information about student learning and experience. While Likert-type items can gauge general levels of student satisfaction, effort, or learning, the rich data of open-ended responses give many more possibilities for characterizing the underlying reasons why students are satisfied or unsatisfied. In circumstances where the data are unwieldy to read, we have shown the ability of the STM to generalize the results of these responses while also incorporating quantitative factors such as student satisfaction. In this way, the STM enables instructors to use course evaluation in a meaningful way, even with short periods between iterations of a course, while lessening the time costs of reading volumes. 
(2015). Computer-Assisted Reading and Discovery for Student-Generated Text in Massive Open Online Courses. Journal of Learning Analytics, 2(1), 156-184.

Topic 1: "Audio-Visual"

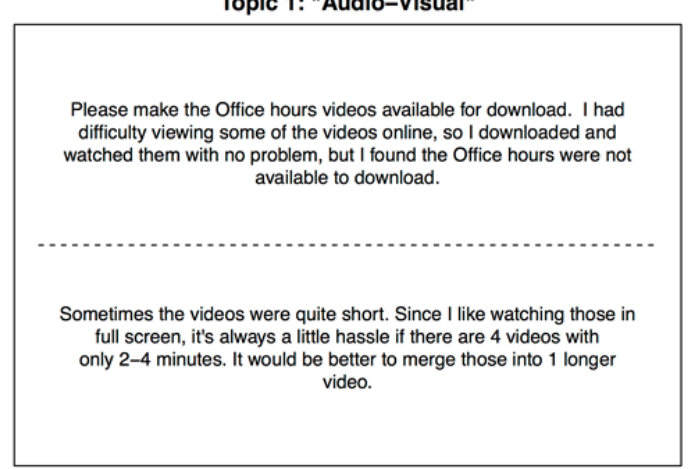

Topic 4: "Assessments"

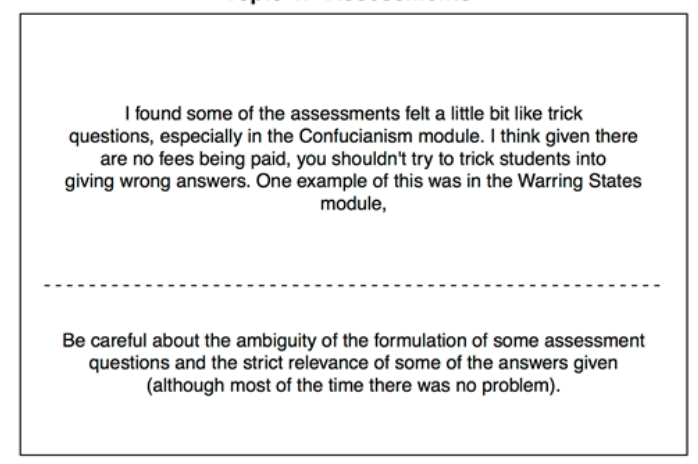

Topic 2: "Discussion Forums"

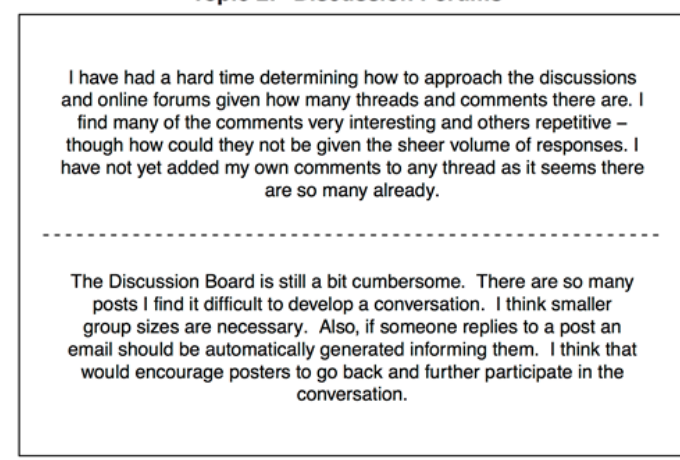

Topic 7: "Course Content"

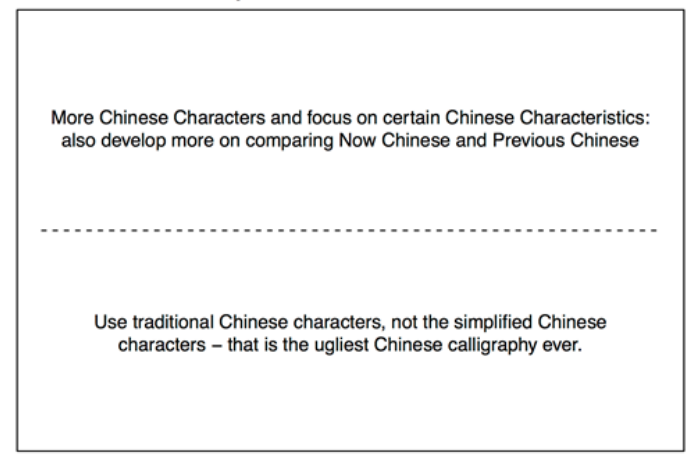

Figure 10. Students discuss what they found could be improved about the class, with examples from topics $1,2,4$, and 7.

\section{CONCLUSION}

As MOOCs and other online learning environments expand in scale, the same data growth that proves overwhelming to faculty and instructional teams increases the reliability and utility of the STM. The STM becomes ever more useful in exactly the place where the human ability to process the entirety of student contributions in a timely fashion breaks down. These computer-assisted reading approaches have promising applications for helping faculty make sense of the vast conversations happening in MOOCs and large-scale learning environments. By way of conclusion, we offer three possible extensions of this work into domains beyond those discussed in this paper.

While the examples in this paper come from discussion forums, pre-course surveys, and course evaluations, there are also applications with student assessment. Much of the early research and development in MOOCs has focused on scalable mechanisms of assessing and assigning grades to individual student work. There have been important advances, but methods of peer grading and machine grading have proven controversial, technically challenging, and logistically difficult to implement (Piech et al., 2013; Rees, 2013). The supervised machine analysis of shorter pieces of student writing has proven particularly intractable (Brew \& Leacock, 2013; Reich et al., 2014a). It may, therefore, 
(2015). Computer-Assisted Reading and Discovery for Student-Generated Text in Massive Open Online Courses. Journal of Learning Analytics, 2(1), 156-184.

prove useful to complement these efforts at individual assessment of student learning with STM and other topic modelling approaches that attempt to assess student learning collectively. STM holds the promise of inviting students to submit their written work, knowing that each of their individual contributions will add to a model of student thinking that represents an entire learning community.

The examples in this paper also exclusively come from MOOCs built within learning management systems, where the focus of learning is on lecture videos and computationally graded assessments (sometimes called $\mathrm{xMOOCS}$ ). STM technologies and methods also hold promise for connectivist learning environments (Downes, 2008), which emphasize the aggregation of student-produced text and media from sites across the open Web (sometimes called cMOOCs). STM approaches to analyzing these aggregated corpora offer connectivist educators a new set of tools to make aggregate meaning of the production of a network of learners. As we look towards a future of learning environments with larger networks of students, the most important technologies will not be those that facilitate dissemination of content from faculty, but those that allow educators to better understand the range and quality of contributions from students.

Finally, we have focused entirely on online environments, but there are promising applications for these tools in residential settings as well. In many large lecture courses, faculty use exit tickets or "mud cards" to have students articulate concepts that they are struggling with, and the STM could help characterize the topics and distributions of those challenges. Course evaluations are another promising domain for future research. Nearly every university uses some form of course evaluation to provide feedback to and evaluate instructors, but for reasons of expediency the analyses of these course evaluations is mostly limited to the quantitative elements. STM models open new possibilities for helping faculty, administrators, and instructional staff better understand not just how satisfied and engaged students were in a course, but how they qualitatively describe the strengths and weaknesses of particular courses.

Throughout higher education and across the disciplines, reasoning from evidence through writing is one of the central ways that students develop and demonstrate their understanding. Structural Topic Models and other unsupervised machine-learning methods are an important set of tools, complementary to peer grading and supervised machine learning techniques, to help instructors and educational researchers better understand students' written contributions to learning communities and learning experiences.

\section{REFERENCES}

Anaya, A. R., \& Boticario, J. G. (2011). Application of machine learning techniques to analyse student interactions and improve the collaboration process. Expert Systems with Applications, 38(2), 1171-1181. doi:10.1016/j.eswa.2010.05.010

Blei, D. M. (2012). Probabilistic topic models. Communications of the ACM, 55(4), 77-84. doi:10.1145/2133806.2133826 
(2015). Computer-Assisted Reading and Discovery for Student-Generated Text in Massive Open Online Courses. Journal of Learning Analytics, 2(1), 156-184.

Blei, D. M., Ng, A., \& Jordan, M. (2003). Latent Dirichlet allocation. Journal of Machine Learning Research, 3, 993-1022. Retrieved from http://machinelearning.wustl.edu/mlpapers/paper files/BleiNJ03.pdf

Bishop, C. M. (2006). Pattern recognition and machine learning. New York, NY: Springer.

Breslow, L., Pritchard, D. E., DeBoer, J., Stump, G. S., \& Ho, A. D. (2013). Studying learning in the worldwide classroom: Research into EdX's first MOOC. Research \& Practice in Assessment, 8(1), 13-25. http://www.rpajournal.com/dev/wp-content/uploads/2013/05/SF2.pdf

Brew, C., \& Leacock, C. (2013). Automated short answer scoring. In M. Shermis \& J. Burnstein (Eds.), Handbook of automated essay evaluation: Current applications and new directions (pp. 136153). New York, NY: Routledge.

Brinton, C. G., Chiang, M., Jain, S., Lam, H., Liu, Z., \& Wong, F. M. F. (2013). Learning about social learning in MOOCs: From statistical analysis to generative model. arXiv preprint arXiv:1312.2159.

Chang, J., Gerrish, S., Wang, C., Boyd-Graber, J. L., \& Blei, D. M. (2009). Reading tea leaves: How humans interpret topic models. In Proceedings of Advances in Neural Information Processing Systems 22 NIPS (2009).http://papers.nips.cc/paper/3700-reading-tea-leaves-how-humans-interpret-topicmodels Computing Research Association. (2013). New technology-based models for postsecondary learning: Conceptual frameworks and research agenda. Retrieved from http://cra.org/uploads/documents/resources/rissues/Postseconday Learning NSFCRA report.pdf

Downes, S. (2008). Places to go: Connectivism \& connective knowledge. Innovate: Journal of Online Education, 5(1). http://bsili.3csn.org/files/2010/06/Places to GoConnectivism Connective Knowledge.pdf

Dringus, L. P., \& Ellis, T. (2005). Using data mining as a strategy for assessing asynchronous discussion forums. Computers \& Education, 45(1), 141-160. doi:10.1016/j.compedu.2004.05.003

Duwairi, Rehab M. (2006). A framework for the computerized assessment of university student essays. Computers in Human Behavior, 22(3), 381-388.

Eggers, A., \& Spirling, A. (2011). Partisan convergence in executive-legislative interactions modeling debates in the House of Commons, 1832-1915. Retrieved from Harvard Institute for Quantitative Social Science website:

202.154.59.182/mfile/files/Jurnal/Jurnal\%202011/Partisan\%20Convergence\%20in\%20Executive $-$

Legislative\%20Interactions\%20Modeling\%20Debates\%20in\%20the\%20House\%20of\%20Commo ns,\%201832\%961915.pdf

Fisher, W. W. (2014). HLS1x: CopyrightX course report. (HarvardX Working Paper Series 5.) Retrieved from SSRN: http://papers.ssrn.com/sol3/papers.cfm?abstract id=2382332

Grainger, B. (2013). Massive open online course (MOOC) report. Retrieved from University of London website: http://www.londoninternational.ac.uk/sites/default/files/documents/mooc_report2013.pdf

Grimmer, J., \& King, G. (2011). General purpose computer-assisted clustering and conceptualization. 
(2015). Computer-Assisted Reading and Discovery for Student-Generated Text in Massive Open Online Courses. Journal of Learning Analytics, 2(1), 156-184.

Proceedings of the National Academy of Sciences, 108(7), 2643-2650. Retrieved from http://www.pnas.org/content/108/7/2643.abstract

Grimmer, J., \& Stewart, B. M. (2013). Text as data: The promise and pitfalls of automatic content analysis methods for political texts. Political Analysis, 21(3), 267-297. Retrieved from http://stanford.edu/ igrimmer/tad2.pdf

Guo, P. J., Kim, J., \& Rubin, R. (2014). How video production affects student engagement: An empirical study of MOOC videos. Proceedings of the First ACM Conference on Learning@ Scale conference, 41-50. Retrieved from http://pgbovine.net/publications/edX-MOOC-video-production-andengagement LAS-2014.pdf

Hastie, T., Tibshirani, R., \& Friedman, J. (2009). The elements of statistical learning (2nd Ed.). New York, NY: Springer.

Hillard, D., Purpura, S., \& Wilkerson, J. (2008). Computer-assisted topic classification for mixed-methods social science research. Journal of Information Technology \& Politics, 4(4), 31-46. Retrieved from http://faculty.washington.edu/jwilker/TopicClassification.pdf

Ho, A. D., Reich, J., Nesterko, S. O., Seaton, D. T., Mullaney, T., Waldo, J., \& Chuang, I. (2014). HarvardX and MITx: The first year of open online courses (HarvardX and MITx Working Paper No. 1). Retrieved from SSRN: http://papers.ssrn.com/sol3/papers.cfm?abstract id=2381263

Jamal, A., Keohane, R., Romney, D., \& Tingley, D. (2014). Anti-Americanism or anti-interventionism: Evidence from the Arabic Twitter universe. Perspectives on Politics, 13(1), 55-73. Retrieved from http://scholar.harvard.edu/dtingley/publications/american-eyes-arabic-tweeters

King, G., Pan, J., \& Roberts, M. E. (2013). How censorship in China allows government criticism but silences collective expression. American Political Science Review, 107, 1-18. Retrieved from http://gking.harvard.edu/files/censored.pdf

Kizilcec, R. F. \& Schneider, E. (2015). Motivation as a lens to understand online learners: Towards a datadriven design with the OLEI scale. Transactions on Computer-Human Interactions, 22(2). doi:10.1145/2699735

Koller, D., Ng, A., Do, C., \& Chen, Z. (2013, May/June). Retention and intention in massive open online courses: In depth. Educause Review. Retrieved from http://net.educause.edu/ir/library/pdf/ERM1337.pdf

Kolowich, S. (2014). The professors who make the MOOCs. The Chronicle of Higher Education, 59(28), A20-A23. Retrieved from http://chronicle.com/article/The-Professors-Behind-the$\mathrm{MOOC} / 137905 / \#$ id=overview

Lowe, W., \& Benoit, K. (2013). Validating estimates of latent traits from textual data using human judgment as a benchmark. Political Analysis, 21(3), 298-313. Retrieved from http://pan.oxfordjournals.org/content/early/2013/06/17/pan.mpt002

Lucas, C., Nielsen, R., Roberts, M., Stewart, B., Storer, A., \& Tingley, D. (2013). Computer assisted text analysis for comparative politics. Retrieved from http://scholar.harvard.edu/files/dtingley/files/comparativepoliticstext.pdf

Mak, S. F. J., Williams, R., \& Mackness, J. (2010). Blogs and forums as communication and learning tools 
(2015). Computer-Assisted Reading and Discovery for Student-Generated Text in Massive Open Online Courses. Journal of Learning Analytics, 2(1), 156-184.

in a MOOC. Proceedings of the 7th International Conference on Networked Learning 2010, 275285. Retrieved from http://www.lancaster.ac.uk/fss/organisations/netlc/past/nlc2010/abstracts/PDFs/Mak.pdf Mimno, D., Wallach, H. M., Talley, E., Leenders, M., \& McCallum, A. (2011, July). Optimizing semantic coherence in topic models. Proceedings of the Conference on Empirical Methods in Natural Language Processing (pp. 262-272). Association for Computational Linguistics. http://dl.acm.org/citation.cfm?id=2145462

Murphy, E., \& Rodriguez-Manzanares, M. A. (2012). Rapport in distance education. The International Review of Research in Open and Distance Learning, 13(1). Retrieved from http://www.irrodl.org/index.php/irrodl/article/view/1057/2076

Murphy, K. P. (2012). Machine learning: A probabilistic perspective. Cambridge, MA: MIT Press.

Piech, C., Huang, J., Chen, Z., Do, C., Ng, A., \& Koller, D. (2013). Tuned models of peer assessment in MOOCs. arXiv preprint arXiv:1307.2579

Quinn, K. M., Monroe, B. L., Colaresi, M., Crespin, M. H., \& Radev, D. R. (2010). How to analyze political attention with minimal assumptions and costs. American Journal of Political Science, 54(1), 209228. Retrieved from https://www.law.berkeley.edu/files/TopicModel.pdf

Team, R. Core (2012). R: A language and environment for statistical computing. Vienna, Austria: R Foundation for Statistical Computing. Retrieved from: http://cran.case.edu/web/packages/dplR/vignettes/timeseries-dplR.pdf

Reich, J., Nesterko, S. O., Seaton, D. T., Mullaney, T., Waldo, J., Chuang, I., \& Ho, A. D. (2014a). PH207x: Health in Numbers and PH278x: Human Health and Global Environmental Change 2012-2013 course report. (HarvardX Working Paper No. 2.) Retrieved from SSRN: http://papers.ssrn.com/sol3/papers.cfm?abstract id=2382242

Reich, J., Emanuel, J., Nesterko, S. O., Seaton, D. T., Mullaney, T., Waldo, J., \& Ho, A. D. (2014b). HeroesX: The Ancient Greek Hero: Spring 2013 course report. (HarvardX Working Paper No. 3.) Retrieved from SSRN: http://papers.ssrn.com/sol3/papers.cfm?abstract id=2382246

Reich, J., Nesterko, S. O., Seaton, D. T., Mullaney, T., Waldo, J., Chuang, I., \& Ho, A. D. (2014c). JusticeX: Spring 2013 course report. (HarvardX Working Paper No. 4.) Retrieved from SSRN: http://papers.ssrn.com/sol3/papers.cfm?abstract id=2382248

Rees, J. (2013, March 5). Peer grading can't work. Inside Higher Ed. Retrieved from http://www.insidehighered.com/views/2013/03/05/essays-flaws-peer-gradingmoocs\#ixzz2MiKxNP7b

Roberts, M. E., Stewart, B. M., \& Airoldi, E. M. (2014). Structural topic models. Working Paper. Retrieved from http://scholar.harvard.edu/files/bstewart/files/stm.pdf

Roberts, M. E., Stewart, B. M., \& Tingley, D. (2014). stm: R package for structural topic models. Working Paper. Retrieved from http://scholar.harvard.edu/files/bstewart/files/stmvignette.pdf

Roberts, M. E., Stewart, B. M., Tingley, D., \& Airoldi, E. M. (2013). The structural topic model and applied social science. Advances in Neural Information Processing Systems Workshop on Topic Models: Computation, Application, and Evaluation. Retrieved from http://scholar.harvard.edu/files/bstewart/files/stmnips2013.pdf 
(2015). Computer-Assisted Reading and Discovery for Student-Generated Text in Massive Open Online Courses. Journal of Learning Analytics, 2(1), 156-184.

Roberts, M. E., Stewart, B. M., Tingley, D., Lucas, C., Leder-Luis, J., Gadarian, S. K., Albertson, B, \& Rand, D. G. (2014). Structural topic models for open-ended survey responses. American Journal of Political Science, 58(4), 1064-1082. doi: 10.1111/ajps.12103

Romero, C., \& Ventura, S. (2007). Educational data mining: A survey from 1995 to 2005. Expert Systems with Applications, 33(1), 135-146. Retrieved from http://www.sciencedirect.com/science/article/pii/S0957417406001266

Shermis, M. D., \& Hamner, B. (2012). Contrasting state-of-the-art automated scoring of essays: Analysis. Paper presented at National Council on Measurement in Education Annual Meeting, Vancouver, B.C. http://www.scoreright.org/NCME_2012_Paper3_29_12.pdf

Stewart, B. M., \& Zhukov, Y. M. (2009). Use of force and civil-military relations in Russia: An automated content analysis. Small Wars \& Insurgencies, 20(2), 319-343. Retrieved from http://scholar.harvard.edu/files/bstewart/files/2009 stewartzhukov swi.pdf

Stockmann, D. (2012). Media commercialization and authoritarian rule in China. Cambridge, UK: Cambridge University Press.

Stump, G. S., DeBoer, J., Whittinghill, J., \& Breslow, L. (2013). Development of a framework to classify MOOC discussion forum posts: Methodology and challenges. Retrieved from https://tll.mit.edu/sites/default/files/library/Coding a MOOC Discussion Forum.pdf

Thomas, M. (2002). Learning within incoherent structures: The space of online discussion forums. Journal of Computer Assisted Learning, 18(3), 351-366. doi/10.1046/j.0266-4909.2002.03800.x

Van Atteveldt, W., Kleinnijenhuis, J., \& Ruigrok, N. (2008). Parsing, semantic networks, and political authority using syntactic analysis to extract semantic relations from Dutch newspaper articles. Political Analysis, 16(4), 428-446. Retrieved from http://pan.oxfordjournals.org/content/16/4/428.short

Wallach, H. M., Murray, I., Salakhutdinov, R., \& Mimno, D. (2009, June). Evaluation methods for topic models. Proceedings of the 26th Annual International Conference on Machine Learning (pp. 1105-1112). New York, NY: ACM Press. http://dl.acm.org/citation.cfm?id=1553515

Wang, Y. \& Baker, R. S. J. d. (2014). MOOC learner motivation and course completion rates. Working Paper. Retrieved from http://www.moocresearch.com/wp-content/uploads/2014/06/MRIReport-WangBaker-June-2014.pdf

Winchester, T. M., \& Winchester, M. K. (2014). A longitudinal investigation of the impact of faculty reflective practices on students' evaluations of teaching. British Journal of Educational Technology, 45(1), 112-124. Retrieved from http://onlinelibrary.wiley.com/doi/10.1111/bjet.12019/abstract

Yang, D., Wen, M., Kumar, A., Xing, E., Rosé, C. P. (2014). Towards an integration of text and graph clustering methods as a lens for studying social interaction in MOOCs. Working paper. Retrieved from http://www.moocresearch.com/wp-content/uploads/2014/06/C9184 ROSE MOOCResearch-InitiativeRose9184X.pdf 
(2015). Computer-Assisted Reading and Discovery for Student-Generated Text in Massive Open Online Courses. Journal of Learning Analytics, 2(1), 156-184.

\section{APPENDIX}

Top Words

\begin{tabular}{|c|}
\hline $\begin{array}{l}\text { Topic 1: } \\
\text { improv, skill, develop, career, profession, person, advanc, self, enrich, job, } \\
\text { appli, knowledg, technolog, life, use, work, goal, chang, area, becom }\end{array}$ \\
\hline $\begin{array}{l}\text { Topic 2: } \\
\text { learn, new, thing, someth, much, love, possibl, want, life, way, can, } \\
\text { technolog, appli, experi, explor, keep, know, use, subject, interest }\end{array}$ \\
\hline $\begin{array}{l}\text { Topic 3: } \\
\text { educ, interest, continu, subject, explor, topic, keep, experi, life, area, } \\
\text { learn, possibl, want, technolog, love, way, new, differ, like, think }\end{array}$ \\
\hline $\begin{array}{l}\text { Topic 4: } \\
\text { knowledg, expand, increas, broaden, job, appli, technolog, area, achiev, } \\
\text { experi, field, improv, enrich, life, get, want, use, engin, advanc, world }\end{array}$ \\
\hline $\begin{array}{l}\text { Topic 5: } \\
\text { gain, enhanc, acquir, knowledg, appli, use, field, experi, skill, technolog, } \\
\text { engin, job, career, life, futur, inform, area, becom, want, advanc }\end{array}$ \\
\hline $\begin{array}{l}\text { Topic 6: } \\
\text { health, research, public, statist, clinic, method, epidemiolog, biostatist, } \\
\text { quantit, appli, use, basic, work, chang, abl, master, improv, understand, } \\
\text { field, student }\end{array}$ \\
\hline $\begin{array}{l}\text { Topic 7: } \\
\text { mput, program, scienc, basic, technolog, engin, languag, becom, appli, learn } \\
\text { want, use, job, get, inform, field, know, skill, good, like }\end{array}$ \\
\hline $\begin{array}{l}\text { Topic 8: } \\
\text { cours, like, onlin, take, opportun, school, class, colleg, complet, time, } \\
\text { great, free, harvard, edx, univers, also, love, experi, student, want }\end{array}$ \\
\hline $\begin{array}{l}\text { Topic 9: } \\
\text { work, will, help, can, edx, use, goal, life, futur, abl, also, way, make, } \\
\text { think, student, hope, appli, job, achiev, much }\end{array}$ \\
\hline $\begin{array}{l}\text { Topic 10: } \\
\text { want, get, world, univers, experi, best, certif, know, good, achiev, harvard, } \\
\text { also, edx, job, engin, way, student, appli, can, possibl }\end{array}$ \\
\hline $\begin{array}{l}\text { Topic 11: } \\
\text { studi, field, degre, area, current, differ, master, inform, chang, engin, } \\
\text { appli, technolog, work, job, want, possibl, like, achiev, interest, also }\end{array}$ \\
\hline $\begin{array}{l}\text { Topic 12: } \\
\text { understand, better, becom, appli, basic, technolog, use, work, achiev, job, } \\
\text { engin, get, abl, life, world, futur, make, scienc, want, goal }\end{array}$ \\
\hline
\end{tabular}

Top Topics

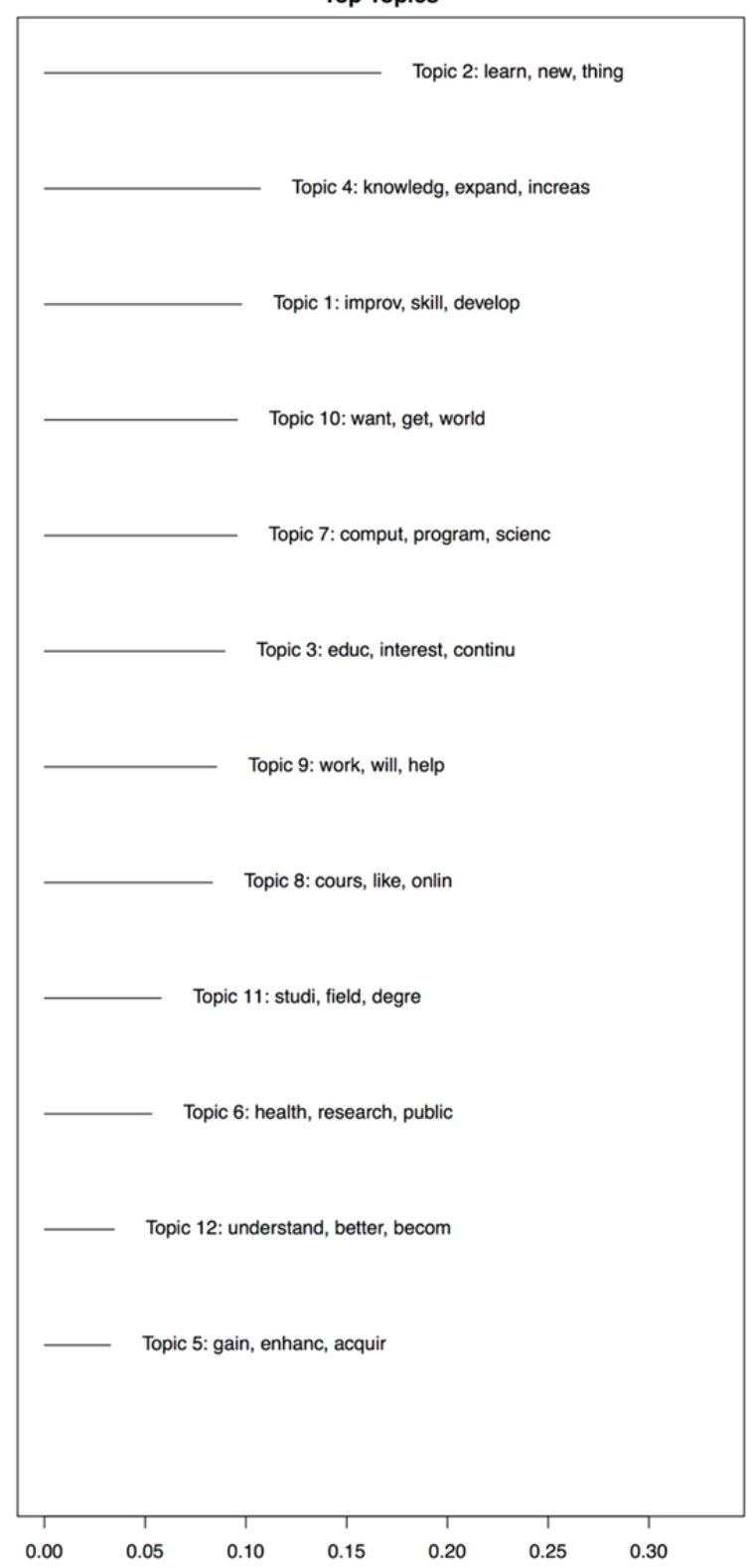

Figure 11. Educational goals. The left column lists words associated with each topic and the right column gives the distribution of topics across the corpus. 